\title{
Interaction between envelope protein of Jaagsiekte Sheep Retrovirus and Hippo signaling pathway is inferred from transcriptome analysis of naturally infected Ovine Pulmonary Adenomatosis
}

\section{XuJie Duan}

Inner Mongolia Agricultural University https://orcid.org/0000-0002-1574-6038

Hui Yang

Inner Mongolia Agricultural University

Liang Zhang

Inner Mongolia Agricultural University

Huiping Li

Inner Mongolia Agricultural University

\section{Zhiwei Zhi Sun}

Inner Mongolia Agricultural University

Xiaoyue Du

Inner Mongolia Agricultural University

Pei Zhang

Inner Mongolia Agricultural University

\section{Sixu Chen}

Inner Mongolia Agricultural University

\section{Ran Liu}

Inner Mongolia Agricultural University

Shuying Liu ( $\sim$ liushuying_imau@126.com )

Inner Mongolia Agricultural University https://orcid.org/0000-0002-5502-9578

\section{Research Article}

Keywords: Ovine pulmonary adenocarcinoma (OPA), Jaagsiekte sheep retro virus 34 (JSRV), transcriptome sequencing analysis (RNA-Seq), Hippo signaling pathway

Posted Date: November 30th, 2021

DOI: https://doi.org/10.21203/rs.3.rs-1103218/v1 
License: (c) (i) This work is licensed under a Creative Commons Attribution 4.0 International License. Read Full License 
Interaction between envelope protein of Jaagsiekte Sheep Retrovirus and Hippo signaling pathway is inferred from transcriptome analysis of naturally infected Ovine Pulmonary Adenomatosis

XuJie Duan', 2,3, Hui Yang 1, 2,3, Liang Zhang ${ }^{1,2,3}$, HuiPing Li1,2,3, ZhiWei Sun ${ }^{1,2,3}$, XiaoYue Du 1, 2,3, Pei Zhang 1, 2,3, SiXu Chen 1, 2, 3, Liu Ran 1, 2, 3, ShuYing Liu 1, 2, 3*

${ }^{1}$ College of Veterinary Medicine of Inner Mongolia Agricultural University, Zhao Wu Da Road 306\#, Hohhot 010018, People's Republic of China

${ }^{2}$ Inner Mongolia Key Laboratory of Basic Veterinary Science, Hohhot 010018, People's Republic of China

${ }^{3}$ Key Laboratory of Clinical Diagnosis and Treatment Technology in Animal Disease, Ministry of Agriculture, Hohhot 010018, People's Republic of China

\section{${ }^{*}$ Corresponding author: ShuYing Liu}

Veterinary Medicine College of Inner Mongolia Agricultural University, Zhao Wu Da Road 306\#, Hohhot 010018, People's Republic of China

E-mail: liushuying_imau@126.com

Tel: +8613039505565 


\section{Abstract}

2 Background: Ovine pulmonary adenomatosis (OPA) is a contagious lung epithelial tumor of sheep caused by jaagsiekte sheep retrovirus (JSRV), which causes severe

4 economic losses for the sheep industry in the world. The specific oncogenic 5 mechanism of JSRV is not yet clarified.

6 Methods: In this study, RNA was extracted from lung tissues of 3 naturally infected

7 OPA cases and 3 healthy individuals for transcriptome sequencing (RNA-Seq).

8 Quantitative reverse transcription-polymerase chain reaction (qRT-PCR) was used to confirm the sequencing data. Immunohistochemistry (IHC) and western blot (WB) were performed to confirm the signaling pathway enriched by DEGs that was activated in naturally infected OPA cases. Cell viability, wound-healing, transwell and colony formation assays were performed to assess the cell malignant transformation of sheep trophoblast cells (STCs) transformed with JSRV-env lentivirus in vitro, and then WB was performed to confirm the signaling pathway that had been validated in the lung tissues.

Results: A total of 366 DEGs (154 up-regulated and 212 down-regulated) were identified by RNA-Seq of lung tissues of naturally infected OPA cases and healthy individuals. GO analysis showed that 366 DEGs were significantly enriched in 178 GO terms, including 114 biological processes, 19 cellular components and 45 molecular functions. KEGG analysis showed that the DEGs mainly enriched in cell proliferation, differentiation, apoptosis and migration, such as PI3K/Akt/mTOR, MAPK and Hippo signaling pathway, and Hippo signaling pathway has never been reported in naturally infected OPA cases. qRT-PCR results of 10 DEGs which were selected randomly were consistent with RNA-Seq results. The protein expression of Hippo signaling pathway were up-regulated in naturally infected OPA lung tissues. 
26 Cell viability, wound-healing, transwell and colony formation assays confirmed that 27 JSRV-env lentivirus caused malignant transformation of STCs and JSRV Env 28 increased the protein expression of Hippo signaling pathway.

29 Conclusions: This research first identified the changes in the transcriptome level of 30 naturally infected OPA lung tissues. These data confirm that the Hippo signaling 31 pathway is involved in the mechanism of OPA, clarify the interaction between Hippo 32 signaling pathway and JSRV Env, provide further evidence for the tumorigenic 33 mechanism of JSRV.

34 Keywords: Ovine pulmonary adenocarcinoma (OPA), Jaagsiekte sheep retrovirus 35 (JSRV), transcriptome sequencing analysis (RNA-Seq), Hippo signaling pathway 


\section{Background}

Ovine pulmonary adenomatosis (OPA), also known as ovine pulmonary adenocarcinoma, jaagsiekte (Afrikaans = driving sickness) and ovine pulmonary carcinoma (OPC), is a contagious lung epithelial tumor disease of sheep [1.2]. Clinical manifestations of the disease include dyspnea, cough and depression, and eventually death due to loss of alveolar function and dyspnea. OPA tumor cells are derived from lung secreting epithelial cells - type II alveolar epithelial cells and clara cells [3]. Its similarities with human bronchioloalveolar adenocarcinoma (BAC) were stressed as early as 1939. The pathological and physiological characteristics of OPA are similar to BAC, both of which present multifocal growth of type II alveolar epithelial cells and clara cell and have relatively well-differentiated tumors. The activation of cellular signal pathways of OPA is also basically similar to BAC [4]. Additionally, advanced stage of naturally infected OPA cases can be regarded as a good model for the pre-clinical research of BAC. The disease occurs widely in the sheep industry all over the world and seriously affects the development of global animal husbandry.

OPA is caused by jaagsiekte sheep retrovirus (JSRV) which belongs to the family Retroviridae, subfamily Orthoretrovirinae, genus Betaretrovirus. The tumorigenicity of JSRV depends on the envelope protein (Env) which is encoded by oncogene-env, mediating malignant transformation of cells [5]. The malignant transformation of cells is mainly regulated by cancer-related signaling pathways, which are usually divided into receptor-dependent and non-receptor-dependent mechanisms. The receptor-dependent mechanism is that the surface protein of JSRV Env recognize and bind to the specific receptor hyaluronidase 2 (Hyal2) of cell surface, then the transmembrane protein of JSRV Env is responsible for the fusion of 
the virus envelope with the cell membrane [6]. Phosphatidylinositol 3'-kinase serine (PI3K)-Threonine kinase B (Akt), Renin-angiotensin system (RAS)/ Mitogen-activated protein kinase (MEK)/Mitogen-activated kinase-like protein (MAPK), Receptor tyrosine kinase (RON) signaling pathway, JSRV stimulates cell proliferation and transforms cells by regulating cell cycle [7.8]. Non-receptor-dependent mechanism means that Env activates PI3K-Akt-mTOR and MAPK signaling pathways through the particular a YXXM motif as a putative docking site of PI3K-Akt-mTOR of the cytoplasmic tail of Env to leads to cell transformation, this process does not need to bind to cell receptor Hyal2 [9.10]. There are complex molecular signal cascade reactions in the tumorigenesis of OPA. To better understand the pathogenesis of JSRV, it is necessary to explore the signal transduction pathways involved in the cell transformation of JSRV Env.

A large number of studies have used the RNA-Seq method to investigate gene expression differences at the RNA level [11]. RNA-Seq is considered to be an effective transcription analysis method, which is more accurate, more replicated and more wider detection range than traditional methods [12.13]. At present, RNA-Seq sequencing technology has been widely used in the study of molecular pathogenesis of hepatitis, AIDS and Brucella [14.15.16]. Karagianni conducted transcriptomic sequencing of lambs artificially infected with JSRV for a short period of time and found 1,971 differential transcripts (1,237 up-regulated, 734 down-regulated), but this data was not applicable to clinically significant adult sheep [17].

Up to now, RNA-Seq sequencing has not been used to reveal transcript differences in naturally infected OPA cases. In order to further study the tumorigenesis mechanism of JSRV, RNA-seq sequencing technology was used in this study to analyze the naturally infected OPA cases and identify the differentially 
expressed genes (DEGs). Through GO and KEGG pathway enrichment analysis, the biological functions of DEGs were analyzed and new signaling pathways related to DEGs enrichment were found. This study provides new research data for the mechanism of JSRV tumorigenesis.

\section{Material and methods}

\section{Ethics statement}

All the protocols of this study involving the use of animals were in accordance with approved Guidelines for Animal Experiments of Inner Mongolia Agricultural University (Approval ID:2020007).

\section{Sample collection}

Three confirmed naturally infected OPA cases (sex, female; age, $2 \pm 0.2$ years; weight, $22.17 \pm 1.12 \mathrm{~kg}$ ) and three healthy sheep (sex, female; age, $2 \pm 0.4$ years; weight, $22.34 \pm 1.45 \mathrm{~kg}$ ) from the Center for Disease Control and Prevention in Inner Mongolia. Lung tissues were collected from sheep and washed in physiological saline for three times to minimize the blood contamination. One portion of each lung tissue sample was fixed with $10 \%$ formalin for immunohistochemistry (IHC) assay, and the rest of each lung tissue sample was immediately plunged into nitrogen canister for further mRNA and protein expression analysis.

\section{RNA extraction, quality control, cDNA synthesis and library preparation}

Total RNA was extracted from the lung tissues of 3 naturally infected OPA individuals and 3 healthy sheep using Trizol reagent (Takara, Dalian, China) according to the manufacturer's instructions. Total RNA degradation and contamination was assessed on $1 \%$ agarose gels, quality and purity were further assessed using the BioAnalyzer 2100 biological analyzer and the RNA 6000 Nano 
LabChip Kit (Agilent, CA, USA). The RNA Integrity Number (RIN) >7.0. Poly-(A) mRNA was isolated from approximately $10 \mu \mathrm{g}$ total RNA by poly-T oligo attached magnetic beads and Oligotex mRNA Kit (Thermo Fisher Scientific, Walthan, MA, USA). Purified mRNA was randomly disrupted by Fragmentation Buffer (Ebricentre, WI, USA) at high temperature, and using these short fragments as templates, the first-strand cDNA was synthesized by reverse transcriptase and random hexamer primers (Sangon Biotech, Shanghai, China). The second-strand cDNA was synthesized by using Buffer, dNTPs, RNase H and DNA Polymerase I (Sangon Biotech, Shanghai, China). The double-strand cDNA fragments were subjected to end repair and adapter ligation. Adapter-modified fragments were selected using AMPure XP beads (Thermo Fisher Scientific, Walthan, MA, USA) and amplified to construct the final cDNA library. Transcriptome sequencing was performed using the high-throughput sequencing platform of Illumina HiSeq 4000 (Illumina, San Diego, CA, USA) and the length of paired-end reads was $300 \mathrm{bp}( \pm 50 \mathrm{bp})$.

\section{Sequencing analyses and bioinformatics analysis}

To ensure the quality and reliability of the data analysis, the quality of the original data was controlled before the data analysis. Data processing prior to assembly, low quality reads (including reads from sequencing adapters; reads with a ratio of $\mathrm{N}>10$; reads with all the $\mathrm{A}$ base; the number of bases of qvalue $\leqslant 20$ accounted for more than $50 \%$ of the whole read) were removed[18]. The clean reads obtained were compared and assembled with the sheep reference genome (version: Ovis_aries_3.1) of UCSC (http://genome.ucsc.edu/) using Hisat (version:2.0). The 
mapped reads from each sample were assembled using StringTie (version 1.3.0: version) and merged into a new transcript, and the transcript expression levels were analyzed using StringTie and Ballgown(version:3.2.5) [19]. Based on the location information of the gene sequence on the reference genome, the FeatureCounts (version:1.5.0-P3) was used to count the number of reads from start to finish of each gene (including the new prediction gene) [20]. The obtained read count value were corrected for sequencing depth and gene length to obtained FPKM value (FPKM, fragments per kilobase of exon model per million mapped fragments). The DEseq2 $\mathrm{R}$ package was used for differential analysis and the standard for the screening of differentially expressed genes (DEGs) of padj $<0.05$ and $\mid \log 2$ (FC) $\mid>1$ (fold change) [21]. Then, the R package of clusterProfiler was used for gene ontology (GO) and Kyoto Encyclopedia of Genes and Genomes (KEGG, Kyoto, Japan) pathway enrichment analysis.[22]

\section{qRT-PCR validation of RNA-Seq data}

To confirm the RNA-Seq data, 10 DEGs randomly selected were verified by qRT-PCR. In this experiment, there were 3 biological replicates per group. Table 1 shows the primer sequences of selected mRNA transcripts and the reference gene $\beta$-actin and GAPDH [23]. qRT-PCR was performed using 7500 Real Time System (version: 2.3.1) (Applied Biosystems, Foster City, CA, USA). The final volume of the reaction product was $20 \mu \mathrm{L}$, including :10 $\mu \mathrm{L}$ TB Green ${ }^{\mathrm{TM}}$ Premix Ex Taq $^{\mathrm{TM}}$ II , $0.4 \mu \mathrm{L}$ ROX Reference Dye II (50X) (TaKaRa, Dalian, China), $2 \mu \mathrm{L}$ cDNA, $0.8 \mu \mathrm{L}$ of 10 $\mu \mathrm{M}$ F/R primer (Sangon Biotech, Shanghai, China), and $6 \mu \mathrm{L}$ RNase-free H2O. All 
amplification procedures involved $95^{\circ} \mathrm{C}$ for $30 \mathrm{~s}$ for 1 cycle, $95^{\circ} \mathrm{C}$ for $5 \mathrm{~s}$ and $60^{\circ} \mathrm{C}$ for $34 \mathrm{~s}$ for 40 cycles. The $2^{-\Delta \Delta \mathrm{CT}}$ method was used to analyze the relative expression of genes in qRT-PCR [24]. The results were the mean and standard deviation of the 3 biological replicates.

\section{Immunohistochemistry assay}

The samples of lung tissue were fixed in $10 \%$ formalin for $72 \mathrm{~h}$ and then embedded in paraffin, the embedded tissues were cut into four- $\mu \mathrm{m}$-thick sections. The paraffinic sections were deparaffinized with xylene and rehydrated in a graded ethanol series. Antigen retrieval was achieved by microwaving $\left(100^{\circ} \mathrm{C}\right)$ in $10 \mathrm{mmoL} / \mathrm{L}$ of sodium citrate buffer (Beyotime, Shanghai, China) at $\mathrm{PH}=6$ for $10 \mathrm{~min}$. After washing in phosphate-buffered saline (PBS) (Maixin, Fujian, China), endogenous peroxidase was quenched in methanol with $3 \%$ hydrogen peroxide for $10 \mathrm{~min}$, and then the sections were blocked in $5 \%$ goat serum for $15 \mathrm{~min}$. The sections were incubated with primary antibody at $4^{\circ} \mathrm{C}$ for $12 \mathrm{~h}$, and negative control were incubated with normal rabbit serum instead of the primary antibody. The horseradish peroxidase-labeled secondary antibody (Thermo Fisher, Waltham, MA) was added to the sections and incubated for $15 \mathrm{~min}$. Immunoreactivity of specific protein was visualized by incubation with chromogen 3,3' diaminobenzidine (DAB, Maixin, Fujian, China). The stained sections were counterstained with hematoxylin (Maixin, Fujian, China), dehydrated in a graded ethanol series and used xylene for transparency. After mounting the coverslips with neutral balsam (Maixin, Fujian, China), and the images were taken using microscope (Nikon, Tokyo, Japan). Image Pro Plus (IPP) (Version: 6.0) (Media Cybernetics, CA, USA) was used to quantify the strength of positive immune signals. Five representative visual fields of high-magnification (400x) were selected from each group, and each visual field was analyzed for three times. The 
intensity of these protein immunoreaction was divided into five grades depending on the percentage of positive cells (percentage scores): $<10 \%$ (0), 10-25\% (1), 25-50\% (2), 50-75 (3), and $>75 \%$ (4). The intensity of staining was divided into four grades (intensity scores): no staining (0), light brown (1), brown (2), and dark brown (3). The overall staining score was determined by the formula: overall scores $=$ percentage score $x$ intensity score [25]. The sources and dilution of the primary antibody were shown in the table2.

\section{Cell culture and lentivirus transfection}

The cell line used was sheep trophoblast cells (STCs) which established by our laboratory [26]. STCs were cultured in DMEM-F12 medium (Gibco, Grand Island, NY, USA) supplemented with $10 \%$ fetal bovine serum (FBS, ExCell Bio, Suzhou, Jiangsu, China), $100 \mu \mathrm{g} / \mathrm{mL}$ streptomycin and $100 \mathrm{IU} / \mathrm{mL}$ penicillin (Gibco, Grand Island, NY, USA) at $37{ }^{\circ} \mathrm{C}$ with $5 \% \mathrm{CO}_{2}$ humidified atmosphere. For stable transfections, the cells were infected with JSRV-env lentivirus that established by our laboratory when the cells reached to $3 \times 10^{5}$ in per six-well plate (Corning, NY, USA) and cultured with antibiotic-free and serum-free medium. The medium was replaced with fresh medium supplemented with $15 \%$ fetal bovine serum after $12 \mathrm{~h}$ and the cells were maintained at $37{ }^{\circ} \mathrm{C}$ with $5 \% \mathrm{CO}_{2}$ humidified atmosphere for $72 \mathrm{~h}$. The images were obtained by using the Confocal Laser Scanning Microscope (CLSM) (Carl-Zeiss, Oberkochen, Germany). Image Pro Plus was used to calculate the number of infected cells through counting signals of red fluorescent protein. A549 cells were cultured with $10 \%$ fetal bovine serum, $100 \mu \mathrm{g} / \mathrm{mL}$ streptomycin and $100 \mathrm{IU} / \mathrm{mL}$ penicillin at $37{ }^{\circ} \mathrm{C}$ with $5 \% \mathrm{CO}_{2}$ humidified atmosphere.

\section{Cell viability assay}

$$
\text { Cell viability was determined by MTT assay }
$$


(3-[4,5-dimethyl-2-thiazolyl]-2,5-diphenyltetrazolium bromide). Briefly, cells were cultured in 96-well plates $\left(2 \times 10^{3}\right.$ cells in every well) in medium supplemented with $10 \%$ FBS. After culturing for $24 \mathrm{~h}, 48 \mathrm{~h}, 72 \mathrm{~h}, 20 \mu \mathrm{L}$ of MTT working solution $(5 \mathrm{mg} / \mathrm{mL})$ (Solarbio, Beijing, China) was added to each well and incubated at $37^{\circ} \mathrm{C}$ with $5 \% \mathrm{CO}_{2}$ humidified atmosphere for $4 \mathrm{~h}$, then the medium was removed and the resultant MTT formazan was solubilized by adding $150 \mu \mathrm{L}$ of DMSO (Solarbio, Beijing, China) per well for 10min at room temperature. The absorbance was assessed at $490 \mathrm{~nm}$ by a microplate reader (Biotek, VT, USA) [27].

\section{Wound-healing assay}

Use a marker pen to draw horizontal lines evenly at $0.5 \mathrm{~cm}$ intervals in each well on the back of the six-well plates. $5 \times 10^{5}$ cells were seeded in 6 -well plates in triplicate for culturing at $37{ }^{\circ} \mathrm{C}$ with $5 \% \mathrm{CO}_{2}$ humidified atmosphere overnight. When the cells were plated in 6-well plates and cultured to achieve $100 \%$ confluency, a sterile pipette was used to create wound on the cell layer, then the cells were washed twice with PBS and added serum-free medium. Continue culturing in incubator at $37{ }^{\circ} \mathrm{C}$ with $5 \% \mathrm{CO}_{2}$ humidified atmosphere. Photographs of the scratch area were taken by microscope at 0h, 24h, 48h [28]. The scratch areas were measured by Image Pro Plus software and cell migration rates were calculated.

\section{Cell invasion assay}

Cell invasion assay was performed by using 24-well transwell chambers with a pore size of $8 \mu \mathrm{m}$ (Corning, NY, USA). Matrigel was pre-spread into the upper chamber. $5 \times 10^{4}$ cells were dispersed in $200 \mu \mathrm{L}$ of serum-free medium and then the suspension was added to the upper chamber. $500 \mu \mathrm{L}$ of medium supplemented with $10 \%$ FBS was added to the lower chamber. Cells were cultured at $37{ }^{\circ} \mathrm{C}$ with $5 \% \mathrm{CO}_{2}$ humidified atmosphere for $24 \mathrm{~h}$. The cells on the upper surface of upper chamber were 
scraped off with cotton swab and the upper chamber was cleaned twice with PBS. The cells on the lower surface of upper chamber were fixed with $4 \%$ formaldehyde and stained with $0.1 \%$ crystal violet for $30 \mathrm{~min}$ [29]. The cells were observed by microscope and counted by Image Pro Plus software.

\section{Colony formation assay}

$1.2 \%$ and $0.7 \%$ soft agar (Sigma, MO, USA) were sterilized and cooled to $40^{\circ} \mathrm{C}$. The mixture that mixed with $3 \mathrm{~mL}$ of $1.2 \%$ soft agar and equal volume of prewarmed 2×DMEM supplemented with $20 \%$ FBS was put into 6-cm cell dish to make base agar. After solidification of base gar, logarithmic growth phase cells were diluted to $1 \times 10^{4}$ cells/ mL with DMEM. $0.2 \mathrm{~mL}$ of cell suspension was taken to the mixture that mixed with $0.7 \%$ soft agar and $2 \times$ DMEM supplemented with $20 \%$ FBS. The mixture was then put into 6-cm cell dish to make top agar. After solidification of top agar, the cell dishes were cultured at $37{ }^{\circ} \mathrm{C}$ with $5 \% \mathrm{CO}_{2}$ humidified atmosphere. The number of colonies were counted by microscope 10 days later [30].

\section{Western blot assay}

According to the instruction of the tissue or cell total protein extraction kit (Sangon Biotech, Shanghai, China), total protein was extracted from the samples of lung and cells. Concentration of total protein was measured by the BCA protein assay kit (Beyotime, Shanghai, China). The prorein samples were denatured by SDS-PAGE sample loading buffer (Beyotime, Shanghai, China) at $100^{\circ} \mathrm{C}$ for $10 \mathrm{~min}$, and placed in $-20^{\circ} \mathrm{C}$ for storage. After equal amounts $(25 \mu \mathrm{g})$ of protein was separated by electrophoresis on $10 \%$ SDS-PAGE, electophoretically transferred to the PVDF membrane (Merck, Darmstadt, Germany) and then blocked with 5\% skim milk (Solarbio, Beijiing, China) for $2 \mathrm{~h}$. The membranes were incubated the primary antibodies at $4^{\circ} \mathrm{C}$ for $12 \mathrm{~h}$. After washing the membrane, horseradish 
peroxidase-conjugated secondary antibody (Beyotime, Shanghai, China) was used and incubated for 90min at room temperature [31]. Protein bands were visualized with the chemiluminescence (ECL, Beyotime, Shanghai, China) and detected by Bio Imaging Systems (Tanon, shanghai, China). The primary antibody used were provided in the table2.

\section{Statistical analysis}

Numerical data were expressed as means \pm standard deviations (SDs) and values of three independent experiments $(n=3)$ with three replicates. The difference between means was analyzed using GraphPad Prism 8 software (GraphPad, La Jolla, CA, USA). Statistical significance was evaluated by one-way analysis of variance (ANOVA) followed by Tukey's multiple-comparisons test or two-way ANOVA with Bonferroni's post-hoc test. In addition, $p<0.05(*), p<0.01(* *)$ or $p<0.001(* * *)$ was considered as statistically significant.

\section{Results}

\section{RNA-Seq data and DEGs analysis}

To further study the interaction mechanism of OPA, RNA-Seq was used to analyze the transcriptome differences between naturally infected OPA cases and healthy individuals. The RNA-Seq of each sample produced raw reads of $4.88 \pm 1.34 \times 10^{7}$, and $4.81 \pm 0.75 \times 10^{7}$ of clean reads were obtained after quality control. More than $85.77 \%$ of the clean reads were mapped to the sheep reference genome. The detailed mapping output were summarized in Table 3. A total of 15,149 sheep genes were identified by RNA-Seq analysis and principal component analysis (PCA) of these genes mapped the differences clearly between OPA groups and healthy groups (Figure 1A). RNA-Seq analysis showed that the lung tissues of naturally 
infected OPA cases compared to healthy sheep had 366 DEGs (154 up-regulated and 212 down-regulated) (padj $<0.05$ and $|\log 2(\mathrm{FC})|>1)$ (Figure 1B). These DEGs corresponded to 2,143 differentially expressed transcripts (1,360 up-regulated and 783 down-regulated), and the cluster diagram of the top 100 differentially expressed transcripts was shown in Figure 1C.

\section{GO analysis of DEGs}

To further reveal the biological functions of these DEGs, 366 DEGs were analyzed by GO analysis. DEGs were significantly enriched in 178 GO terms (padj<0.05), including 114 biological process (BP), 19 cellular component (CC) and 45 molecular function (MF) terms. The correlation coefficients of top 20 of GO functional enrichment factors were screened out (Figure 2A). In the BP category, DEGs were mainly enriched in the following biological processes: nucleoside metabolic process (ENSOARG00000014411, UPRT), postreplication repair (POLH, UBE2N), convergent extension involved in gastrulation (NPHP3) and branched-chain amino acid transport (SLC38A7) which relate to cell proliferation, differentiation oncogenic signaling transduction. In the CC category, DEGs were mainly enriched in mast cell granule (RASGRP1, SRGN), filopodium (ACTA1, ENSOARG00000015844, RDX), cortical actin cytoskeleton (MED28, RDX) and Nem1-Spo7 phosphatase complex (CNEP1R1) which involve in the invasion of cancer cells. In the MF category, DEGs were mainly enriched in growth factor activity (FGF11, FGF18, INHA, PGF, TGFB3), Rho guanyl-nucleotide exchange factor activity (ARHGEF37, DNMBP, FGD1, RGL2), guanyl-nucleotide exchange 
300

301

302

303

304

305

306

307

308

309

310

311

312

313

314

315

316

317

318

319

320

321

322

factor activity (DNMBP, FGD1, RASGRP1, RGL2) and L-leucine transmembrane transporter activity (SLC38A7), which involved in the cancer susceptibility, metastasis of cancer cells and tumorigenesis.

KEGG pathway analysis of DEGs

To further analyze the relevant biological functions of DEGs, all DEGs were functionally classified according to KEGG pathway analysis. The highly abundant KEGG pathways which were mainly regulated cell proliferation, differentiation, apoptosis and migration, such as PI3K/Akt/mTOR (ENSOARG00000001262, FGF11, GHR, TSC1, YWHAZ), MAPK (FGF11, MAP3K13, RASGRP1, TGFB3), Hippo (BTRC, TGFB3, YWHAZ) and Cell cycle (DBF4, TGFB3 and YWHAZ) signaling pathways (Table 4). The top 20 KEGG pathways with the highest enrichment correlation coefficients were listed in Figure 2B. These results indicated that these signaling pathways may play an important role in the development of OPA.

RNA-Seq data validation by qRT-PCR

To confirm the RNA-Seq data, 10 DEGs involved in tumorigenesis were randomly selected for RNA-Seq data validation (Figure 3). The relative expression results of qRT-PCR showed that the $\log 2(\mathrm{FC})$ of 10 DEGs were between -3.84 and 2.54 ( $\mathrm{p}<0.05)$. In RNA-Seq results, UBE2N, YWHAZ, MYH15 and FAM180B were down-regulated $(\log 2(\mathrm{FC})<-1.29)(\mathrm{p}<0.05)$ and INHBA, TSPO, CYB561D1, PON2 TAPBP, and ZFAND5 were up-regulated in naturally infected OPA cases (log2 (FC) > 1.13) $(\mathrm{p}<0.05)$. Collectively, qRT-PCR results of 10 DEGs were consistent with RNA-Seq results and confirmed the reliability of RNA-Seq data.

Expression of Hippo signaling pathway components in lung tissues of healthy 


\section{sheep and naturally infected OPA cases}

The localization of the Hippo signaling pathway components (MST1/2, LATS/2 YAP1 and p-YAP1) was assessed in the lung tissues of healthy sheep and naturally infected OPA cases. As shown in Fig. 4A, negative controls (no primary antibody) were negative for staining (Figure 4Aa and $4 \mathrm{Ab}$ ). In the lung tissues of healthy sheep, the expression of JSRV Env had no positive signal (Figure 4Ac). MST1/2 was mainly localized in cytoplasm of alveolar epithelial cells and bronchial epithelial cells (Figure 4Ae). LATS1/2 was weakly positive in cytoplasm of alveolar epithelial cells (Figure 4Ag). In addition, both YAP1 and p-YAP1 were weakly expressed and mainly localized in cytoplasm of alveolar epithelial cells (Figure 4Ai and 4Ak).

In the lung of naturally infected OPA cases, JSRV Env was mainly localized in neoplastic foci that emanated from type II alveolar epithelial cells, forming papillary proliferations (Figure 4Ad), and these cases can be identified as typical cases of OPA. MST1/2 was mainly localized in cytoplasm of hyperplastic type II alveolar epithelial cells (Figure 4Af). Moderate expression of LATS1/2 was also detected in cytoplasm of hyperplastic type II alveolar epithelial cells (Figure 4Ah). YAP1 was strongly positive in nucleus and cytoplasm of hyperplastic type II alveolar epithelial cells, and nuclear labeling was more intense than the cytoplasm labeling (Figure 4Aj). The phosphorylated YAP1 was predominantly detected in the cytoplasm of hyperplastic type II alveolar epithelial cells (Figure 4Al).

The results of the overall staining score showed that the expression of MST1/2, LATS1/2 and p-YAP1 in lung tissues of OPA were significantly higher than that in lung tissues of healthy sheep $(p<0.05)$, the expression of YAP in OPA lung tissues was extremely significantly higher than that in lung tissues of healthy sheep $(p<0.01)$ (Figure 4B). Taken together, the Hippo signaling pathway may regulate OPA tumor 
development.

In order to investigate the relative protein expression of the Hippo signaling pathway components (MST1/2, LATS1/2, YAP1 and p-YAP1) in lung tissues of healthy sheep and naturally infected OPA cases. As shown in Fig. 4C, JSRV Env was only expressed in lung tissues of OPA. The results showed that higher expression levels of Hippo signaling pathway components in the lung tissues of OPA cases compared with the lung tissues of healthy sheep. The protein levels of MST1/2 $(p<0.05), \quad$ LATS1/2 $(p<0.01), \quad$ YAP1 $(p<0.01) \quad$ and phosphorylated YAP1 $(p<0.05)$ were significantly increased in OPA cases. The $\mathrm{p}$-YAP1/YAP1 ratio decreased but not significantly (Fig. 4D).

\section{Expression of Hippo signaling pathway components in STCs and JSRV} Env-transformed STCs

To detect the expression of Hippo signaling pathway components in vitro and further explore whether the significant upregulation of Hippo signaling pathway components in OPA cases is caused by JSRV-env. It is necessary to build an effective cell model of JSRV Env-induced cell malignant transformation, then the relevant biological characteristics in JSRV Env-transformed STCs were detected and the expression of Hippo signaling pathway components in this cell model were detected by WB.

A large number of signals of red fluorescent protein were observed under the CLSM (Fig. 5A) and efficiency of cell infection was accounted for about 80\% (Fig. 5B), which proved that STCs were successfully infected with JSRV-env lentivirus. Firstly, we aimed to investigate whether JSRV Env could affect the proliferation of STCs. MTT assay was performed to assess JSRV Env on the proliferation of STCs. As a result, the growth rates of STCs with stable JSRV Env expression and A549 cells as 
positive control were significantly higher when compared to negative control and blank control (Fig.5C). Therefore, we concluded that JSRV Env promoted the proliferation of STCs. Secondly, we aimed to investigate whether JSRV Env can play a role in the migration and invasion of STCs, wound healing and transwell assays were performed to assess the metastatic ability of JSRV Env-transformed STCs. As shown in Fig.5D, JSRV Env enhanced STCs motility was observed using wound healing assay, the scratch areas of JSRV Env-transformed STCs and A549 cells as positive control were significantly reduced compared with negative control and blank control (Fig.5E). To compare with negative control and blank control, JSRV Env remarkably promoted the migratory and invasive capacities of STCs (Fig.5F and 5G). Finally, colony formation ability was performed to assess malignant transformation of cells. The results indicated that it displayed strong colony formation ability and cell independence in JSRV Env-transformed STCs (Fig.5H). These results revealed the high degree of malignant transformation of JSRV Env-transformed STCs which was similar to that of A549 cells.

In order to investigate the expression of the Hippo signaling pathway components (MST1/2, LATS1/2, YAP1 and p-YAP1) in JSRV Env-transformed STCs (Fig.5I). JSRV Env was only expressed in STCs which was transformed by JSRV-env lentivirus. The protein levels of MST1/2( $p<0.05), \operatorname{LATS} 1 / 2(p<0.05), \operatorname{YAP} 1(p<0.01)$ and phosphorylated YAP1 $(p<0.05)$ were significantly increased in JSRV Env transformed STCs compared with the negative control and blank control. The p-YAP1/YAP1 ratio decreased but not significantly (Fig.5J). All the above results indicated that JSRV Env activates the Hippo signaling pathway to regulate development of OPA.

\section{Discussion}



complex molecular signal interaction. In recent years, it has become very common to understand the molecular mechanism of diseases through RNA-Seq, and a large number of RNA-Seq related studies focus on searching for drug therapeutic targets and disease biological markers [21.23.32]. In this study, lung tissues of healthy sheep and naturally infected OPA cases were collected and DEGs were screened by RNA-Seq. DEGs were analyzed through GO analysis and KEGG pathway analysis. related to DEGs enrichment were sought. This study provided a lot of gene expression information, basic data and direction for further study for the molecular pathogenic mechanism of natural infected OPA at the transcriptomic level. All the research methods used were in accord the standards of analysis and process of the transcriptome data in this experiment. DEGs were mainly concentrated in the biological processes of tumorigenesis, cancer cell invasion in naturally infected OPA lung tissues [33]. We speculated that the genomic stability was disrupted by the unbalanced cellular environment and metabolic stress in the early stage of JSRV infection [34]. Metabolic stress leaded to a 416 large accumulation of misfolded proteins, produced excessive ROS and damaged 417 organelles which further infected DNA synthesis and repair [35]. Tumor cells were filled in lung tissues which leaded to hypoxia when OPA tumor formed [36]. HIF-1 $\alpha$ as the hypoxia -inducible factor further activated the transcription of FGF11, FGF18, 
420

421

INH4 and PGF [37]. Additionally, biological processes such as NADH pyrophosphatase activity, NAD+ diphosphatase activity and DNA-directed DNA polymerase activity provided substrates and energy to tumor cells for growing [38].

A total of 2,143 differential transcripts identified in this experiment were basically consistent with the 1,971 differential transcripts from RNA-Seq of the lambs artificially infected with JSRV from $66 \mathrm{~d}$ to $85 \mathrm{~d}$ identified by Karagianni [17]. The differential transcripts mainly involved in genomic stability (POLH, UPRT, SETBP1), proliferation and differentiation (MPP2, FGF11, TAX1BP1) and oncogenic signaling transduction (INHBA, FGD1, FGF11). The additional transcripts compared with the study of Karagianni's were mainly related to tumorigenesis (SLC38A7, NDUFS1, NUDT12) and energy metabolism (POLH, UBE2N, UPRT). It further indicated that the degree of lung tumorigenesis of naturally infected OPA cases were more serious than that of artificially infected lambs for a short period of time, and this is also the reason for the differential expression of transcription level.

For the complex signaling transduction pathways involved in the tumorigenesis of naturally infected OPA cases, the classical signaling pathways that have been reported include PI3K/Akt/mTOR and MAPK signaling pathway [39]. Sun discovered the phosphorylated forms of PI3K/Akt/mTOR and MAPK signaling pathway through IHC and WB techniques from naturally infected OPA cases, and demonstrated that the PI3K/Akt/mTOR and MAPK signaling pathway were crucial in the development of OPA. The study also identified new marker genes involved in these signaling pathways, such as ENSOARG00000001262, FGF11, GHR, TSC1 and 
442

443

444

445

446

447

448

449

450

451

452

453

454

455

456

457

458

459

460

461

462

463

YWHAZ in PI3K/Akt/mTOR signaling pathway and FGF11, MAP3K13, RASGRP1,

TGFB3 in MAPK signaling pathway. These discovered genes were also important in cancer progression. For example, GHR stimulates cell growth and division and it is related to cancer progression, the high expression of FGF11 is associated with lung cancer [40, 41]. In addition, Hippo signaling pathway has never been reported in naturally infected OPA and the downstream processes of Hippo signaling pathway mainly include cell cycle (YWHAZ, DBF4, FGFß3), growth factor activity (FGF11, FGF18, INHA, PGF, TGFB3) and post-replication repair (POLH, UBE2N).

The Hippo signaling pathway was first defined in Drosophila by genetic mosaic screens [42.43] and it has been shown to control organ size, regulate tissue regeneration and cell contact inhibition by regulating both cell proliferation and apoptosis [44.45]. Numerous studies have shown that the core components of this pathway are conserved throughout evolution [46]. In mammals, Hippo signaling pathway consists of a kinase cascade of mammalian STE20-like 1/2 (MST1/2) and large tumor suppressor 1/2 (LATS1/2). MST forms a heterodimer with the adaptor protein Salvador 1 (SAV1), which enhances MST kinase activity and facilitates MST-LATS interaction [47]. Mob1 homolog (MOB1) and LATS are subsequently phosphorylated by MST and phosphorylated MOB1 binds to the autoinhibitory region of LATS, enabling LATS phosphorylation and activation [48,49]. LATS then phosphorylates Yes-associated protein (YAP), a major effector and transcriptional co-activators which lacks DNA-binding activity [50]. Phosphorylated YAP is sequestered in the cytoplasm by binding to 14-3-3 proteins and then ubiquitinated and 
degraded.[51]. Unphosphorylated or dephosphorylated YAP initiates transcription and induces gene expression by interacting with DNA-binding transcription factor TEA domain family member 1-4 (TEAD 1-4) in the nucleus [52]. Knockdown of TEADs or disruption of the YAP-TEAD interaction disrupts YAP-dependent gene transcription and reduces YAP-induced cell proliferation, oncogenic transformation and the epithelial-to-mesen-chymal transition (EMT) significantly [53]. YAP also interacts with other transcription factors including SMAD family members (Smad), Paired box 3 (Pax3) and T-box transcription factor 5 (TBX5). However, the roles of these transcription factors in mediating the growth-promoting activity of YAP have not been determined [54]. YAP is highly expressed in non-small cell lung cancer (NSCLC) in human, and knockdown of YAP sufficiently suppress proliferation, invasion, and tumor growth in mouse. High expression of YAP is correlated with advanced cancer, lymph node metastasis, and reduced survival [55]. In the study of YAP in lung adenocarcinoma (LUAD), the expression profiling of YAP from GEO and TCGA databases was obtained and the results showed that YAP was significantly highly expressed in LUAD patient samples and associated with 5-year survival. [56]. Overexpression and nuclear localization of YAP have been revealed in common human solid cancer types, for instance, lung, hepatocellular, pancreatic, ovarian, colorectal and prostate carcinomas [57.58]. Dawang Zhou showed that YAP1 was almost entirely cytoplasmic in wild-type liver and almost entirely nuclear in hepatocellular carcinoma (HCC) in cell fractionation experiments [59]. YAP is considered as an independent prognostic indicator of disease-free survival and overall 
survival of HCC patients [60]. In this study, YAP has strong nuclear localization and significantly high expression in the lung of naturally infected OPA cases and highly expressed in JSRV Env transformed cells as well, indicating that YAP may play an important role in the malignant transformation of cells induced by JSRV Env. Karagianni found up-regulation of anterior gradient 2 (AGR2) in artificially infected OPA lambs, and AGR2 has been shown to stimulate expression of the epidermal growth factor (EGF) receptor (EGFR) ligand amphiregulin (AREG) in adenocarcinoma cells [61]. Due to the process that AREG is activated by AGR2 is mediated by YAP1, and YAP1 is the nuclear effector of Hippo signaling pathway. It is speculated that Hippo signaling pathway may be involved in the development of OPA. The IHC detection of Hippo signaling pathway showed that the core components expressed strongly positive signals in the nucleus and cytoplasm. These results indicate that there may be false positive signals and it is necessary to detect the localization of lung in naturally infected OPA tissues [17]. The results of this study showed that MST1/2, LATS1/2 and p-YAP1 were all localized in the cytoplasm, and YAP1 was localized both in the nucleus and cytoplasm. These results were consistent with the study of Steinhardt, indicating that there was little nuclear localization of YAP1 in healthy cells [62]. The relative expression of Hippo signaling pathway proteins in JSRV Env transformed STCs were also significantly higher than those in the blank control and the negative control. The reason of JSRV-env lentivirus we used was that lentivirus infection is more stable than plasmid transfection and its long terminal repeats (LTR) sequence has regulatory elements such as promoters and 
enhancers that improve the expression of virus genes [63]. The cell model of STCs, a secretory epithelial cell derived from sheep, which greatly restore the conditions for JSRV Env infecting in vivo [64.65]. These results indicate that the Hippo signaling pathway was activated by JSRV Env both in vitro and in vivo, confirmed to be involved in the development of OPA.

In this study, the upstream components of YAP were also highly expressed in lung tissues of naturally infected OPA cases and JSRV Env transformed cells. It has been commonly known that MST1/2 and LATS1/2 are tumor-inhibiting factor, and the inactivation of Hippo signaling pathway can lead to the overexpression of YAP. Therefore, we speculate that overexpression of YAP induced feedback mechanism regulates Hippo pathway homeostasis, it may be the reason of the expression of the highly expressed upstream components of YAP [66.67]. Toshiro Moroishi found that LATS1/2 gene knockout in tumor cells significantly inhibited tumor growth in vivo in multiple types of cancer with different host backgrounds [68], that may be the reason for the significant increase of LATS1/2 in this study.

The Hippo signaling pathway is regulated by mechanical environment, $G$ protein coupled receptor signal, level of cell energy, oxidative stress, hypoxia and other signals [69]. The specific mechanism that JSRV Env activates the Hippo signaling pathway is unclear. It may be that JSRV Env directly activates the Hippo signaling pathway through certain receptors, or that the Hippo signaling pathway is regulated by many factors which are related to other signaling pathways, these processes indirectly activate the Hippo signaling pathway from an extremely complex signaling 
network. It is also possible that the malignant proliferation of cancer cells causes cell hypoxia and activates the Hippo signaling pathway. We may reveal the specific mechanisms that the Hippo signaling pathway is activated in OPA and how it is co-regulated with other signaling pathways in future research.

Up to now, no studies have been reported to analyze the molecular mechanism of naturally infected OPA lung tissue by RNA-Seq. In this study, bioinformatic analysis was first used to obtain DEGs in lung tissues of naturally infected OPA cases and healthy sheep, and GO analysis and KEGG analysis were performed for DEGs. We found that JSRV Env activates the Hippo signaling pathway which regulates the development of OPA. The results of this study provide basic data and direction for further research into the complex mechanisms of OPA pathogenicity, provide new ideas and strategies for the study of the pathogenesis of OPA and provide a reliable model for the pre-clinical research of BAC.

\section{Conclusions}

This research first identified the changes in the transcriptome level of naturally infected OPA lung tissues. A total of 366 DEGs (154 up-regulated and 212 down-regulated) were identified by RNA-Seq of lung tissues of naturally infected OPA cases and healthy individuals. GO analysis showed that 366 DEGs were significantly enriched in $178 \mathrm{GO}$ terms, including 114 biological processes, 19 cellular components and 45 molecular functions. BP category mainly affecting genomic stability, oncogenesis and oncogenic signal transductions. CC category mainly involved in the invasion of cancer cells. MF category mainly involved in cell 
552 proliferation, survival, differentiation and migration. KEGG analysis indicated that

553 the signaling pathway DEGs enriched mainly regulated cell proliferation,

554 differentiation, apoptosis and migration, such as PI3K/Akt/mTOR, MAPK and Hippo

555 signaling pathways, and Hippo signaling pathway has never been reported in naturally

556 infected OPA cases. qRT-PCR results of 10 DEGs which were selected randomly were

557 consistent with RNA-Seq results. The proteins expression of Hippo signaling pathway

558 were up-regulated in naturally infected OPA lung tissues. Cell viability,

559 wound-healing, transwell and colony formation assays confirmed that STCs

560 transformed with JSRV-env lentivirus had similar malignant transformation ability to

561 A549, and the proteins expression of Hippo signaling pathway were also up-regulated

562 in JSRV Env transformed STCs. Finally, our study identified that JSRV Env activates

563 the Hippo signaling pathway to regulate development of OPA. Therefore, the above

564 results provide new data and direction for studying OPA and clarify the interaction

565 between Hippo signaling pathway and JSRV Env, and provide further evidence for the

566 tumorigenic mechanism of JSRV.

\section{Abbreviations}

568 OPA:Ovine pulmonary adenomatosis; JSRV: Jaagsiekte sheep retrovirus; IHC:

569 Immunohistochemistry; BAC: Bronchioloalveolar adenocarcinoma; GO: Gene

570 Ontology; KEGG: Kyoto Encyclopedia of Genes and Genomes; BSA: Bovine serum

571 albumin; STCs: Sheep trophoblast cells; Env: Envelope protein; Hyal2:

572 Hyaluronidase 2; PI3K: Phosphatidylinositol 3'-kinase serine; RAS:

573 Renin-angiotensin system; MEK: Mitogen-activated protein kinase; MAPK: 
Mitogen-activated kinase-like protein; AIDS: Acquired immunodefciency syndrome; DEGs: differentially expressed genes; BSA: Bovine serum albumin; EMT: epithelial-to-mesen-chymal transition; LUAD: adenocarcinoma; HCC: hepatocellular carcinoma; AGR2: anterior gradient 2; EGF: epidermal growth factor; LTR: long terminal repeats.

\section{Supplementary Information}

Supplementary materials for this article can be found in the attached file.

\section{Acknowledgements}

We are grateful to Mr. YuLin Ding, Miss. YuanYuan Zhang, Miss. Kai Zhang and Miss. JiaMin Zhao for their help in the experiment.

\section{Authors' contributions}

XD and HY carried out the experiments, obtained and analyzed the data. XD drafted the manuscript. HY and XD performed bioinformatics analysis. XD revised the manuscript. SL (Shuying Liu) designed the project, revised the manuscript and provided financial support. All authors have read and agreed to the published version of the manuscript.

\section{Funding}

This work was supported by the National Natural Science Foundation of China (Grant No. 31760721 and Grant No. 32072819), the Inner Mongolia Grassland Innovative Talent Team Project (Grant No. 20151031), Inner Mongolia Applied Research Project (Grant No.2019GG240).

\section{Availability of data and materials}

The datasets supporting the conclusions of this article were included within the article and its additional files. 
598

599

600

601

602

603

604

605

606

607

608

609

610

611

612

613

614

615

616

617

618

619

620

621

622

623

624

625

626

627

\section{Declarations}

\section{Ethics approval and consent to participate}

All protocols for animal experiments were reviewed and approved by the Animal Care and Use Committee of Inner Mongolia Agricultural University (Hohhot, China).

\section{Consent for publication}

Not applicable.

\section{Competing interests}

The authors declare that they have no competing interests.

\section{Author details}

${ }^{1}$ College of Veterinary Medicine of Inner Mongolia Agricultural University, Zhao Wu Da Road 306\#, Hohhot 010018, People's Republic of China. ${ }^{2}$ Inner Mongolia Key Laboratory of Basic Veterinary Science, Hohhot 010018, People's Republic of China.

${ }^{3}$ Key Laboratory of Clinical Diagnosis and Treatment Technology in Animal Disease, Ministry of Agriculture, Hohhot 010018, People’s Republic of China.

\section{References}

1. York DF, Querat G. A history of ovine pulmonary adenocarcinoma (jaagsiekte) and experiments leading to the deduction of the JSRV nucleotide sequence. Curr Top Microbiol Immunol. 2003; 275:1-23. https://doi.org/10.1007/978-3-642-55638-8_1.

2. Palmarini M, Sharp JM, De las Heras M, Fan H. Jaagsiekte sheep retrovirus is necessary and sufficient to induce a contagious lung cancer in sheep. J Virol. 1999;73(8):6964-7692. https://doi.org/10.1128/JVI.73.8.6964-6972.1999.

3. Palmarini M, Dewar P, De las Heras M, Inglis NF, Dalziel RG, Sharp JM. Epithelial tumour cells in the lungs of sheep with pulmonary adenomatosis are major sites of replication for Jaagsiekte retrovirus. J Gen Virol. 1995;76 (11):2731-2737. https://doi.org/10.1099/0022-1317-76-11-2731.

4. Gray ME, Meehan J, Sullivan P, Marland JRK, Greenhalgh SN, Gregson R, Clutton RE, Ward C, Cousens C, Griffiths DJ, et al. Ovine Pulmonary Adenocarcinoma: A Unique Model to Improve Lung Cancer Research. Front Oncol. 2019; 9:335. https://doi.org/10.3389/fonc.2019.00335. 
5. Liu SL, Miller AD. Oncogenic transformation by the jaagsiekte sheep retrovirus envelope protein. Oncogene. 2007;26(6):789-801. https://doi.org/10.1038/sj.onc.1209850.

6. Vigdorovich V, Strong RK, Miller AD. Expression and characterization of a soluble active form of the jaagsiekte sheep retrovirus receptor Hyal2. J Virol. 2005;79(1):79-86. https://doi.org/10.1128/JVI.79.1.79-86.2005

7. Danilkovitch-Miagkova A, Duh FM, Kuzmin I, Angeloni D, Liu SL, Miller AD, Lerman MI. Hyaluronidase 2 negatively regulates RON receptor tyrosine kinase and mediates transformation of epithelial cells by jaagsiekte sheep retrovirus. Proc Natl Acad Sci U S A. 2003;100(8):4580-4585. https://doi.org/10.1073/pnas.0837136100.

8. Sun X, Du F, Liu S. Modulation of autophagy in exJSRV-env-transfected cells through the Akt/mTOR and MAPK signaling pathway. Biochem Biophys Res Commun. 2017;485(3):672-678. https://doi.org/ 10.1016/j.bbrc.2017.02.099.

9. Palmarini M, Maeda N, Murgia C, De-Fraja C, Hofacre A, Fan H. A phosphatidylinositol 3-kinase docking site in the cytoplasmic tail of the Jaagsiekte sheep retrovirus transmembrane protein is essential for envelope-induced transformation of NIH 3T3 cells. J Virol. 2001;75(22):11002-11009. https://doi.org/10.1128/JVI.75.22.11002-11009.2001.

10. Alberti A, Murgia C, Liu SL, Mura M, Cousens C, Sharp M, Miller AD, Palmarini M. Envelope-induced cell transformation by ovine betaretroviruses. J Virol. 2002;76(11):5387-5394. https://doi.org/10.1128/jvi.76.11.5387-5394.2002.

11. Chen XG, Jiang X, Gu J, Xu M, Wu Y, Deng Y, Zhang C, Bonizzoni M, Dermauw $\mathrm{W}$, Vontas J, et al. Genome sequence of the Asian Tiger mosquito Aedes albopictus reveals insights into its biology genetics and evolution. Proc Natl Acad Sci U S A. 2015;112(44):E5907-5915. https://doi.org/10.1073/pnas.1516410112.

12. Hrdlickova R, Toloue M, Tian B. RNA-Seq methods for transcriptome analysis. Wiley Interdiscip Rev RNA. 2017;8(1): 1364-1381. https://doi.org/10.1002/wrna.1364.

13. Limbach PA, Paulines MJ. Going global: the new era of mapping modifications in RNA. Wiley Interdiscip Rev RNA. 2017;8(1): 1367-1384.

https://doi.org/10.1002/wrna.1367.

14. Affò S, Dominguez M, Lozano JJ, Sancho-Bru P, Rodrigo-Torres D, Morales-Ibanez O, Moreno M, Millán C, Loaeza del Castillo A, et al. Transcriptome analysis identifies TNF superfamily receptors as potential therapeutic targets in alcoholic hepatitis. Gut. 2013;62(3):452-460. https://doi.org/10.1136/gutjnl-2011-301146.

15. Rausell A, Muñoz M, Martinez R, Roger T, Telenti A, Ciuffi A. Innate immune defects in HIV permissive cell lines. Retrovirology. 2016 Jun 27;13(1):43. https://doi.org/10.1186/s12977-016-0275-8.

16. Salmon DM, Zahavi T, Kornspan D. Transcriptomic Analysis of the Brucella melitensis Rev.1 Vaccine Strain in an Acidic Environment: Insights Into Virulence Attenuation. Front Microbiol. 2019;10:250. https://doi.org/10.3389/fmicb.2019.00250. 
17. Karagianni AE, Vasoya D, Finlayson J, Martineau HM, Wood AR, Cousens C, Dagleish MP, Watson M, Griffiths DJ. Transcriptional Response of Ovine Lung to Infection with Jaagsiekte Sheep Retrovirus. J Virol. 2019;93(21):e00876-19. https://doi.org/ 10.1128/JVI.00876-19.

18. Mortazavi A, Williams BA, McCue K, Schaeffer L, Wold B. Mapping and quantifying mammalian transcriptomes by RNA-Seq. Nat Methods. 2008;5(7):621-628. https://doi.org/10.1038/nmeth.1226.

19. Pertea M, Pertea GM, Antonescu CM, Chang TC, Mendell JT, Salzberg SL. StringTie enables improved reconstruction of a transcriptome from RNA-seq reads. Nat Biotechnol. 2015 ;33(3):290-295. https://doi.org/10.1038/nbt.3122.

20. Liao Y, Smyth GK, Shi W. FeatureCounts: an efficient general purpose program for assigning sequence reads to genomic features. Bioinformatics. 2014;30(7):923-930. https://doi.org/10.1093/bioinformatics/btt656.

21. McCarthy DJ, Chen Y, Smyth GK. Differential expression analysis of multifactor RNA-Seq experiments with respect to biological variation. Nucleic Acids Res. 2012;40(10):4288-4297. https://doi.org/10.1093/nar/gks042.

22. Yu G, Wang LG, Han Y, He QY. clusterProfiler: an R package for comparing biological themes among gene clusters. OMICS. 2012;16(5):284-287. https://doi.org/10.1089/omi.2011.0118.

23. Yang H, Zhang L, Liu S. Determination of reference genes for ovine pulmonary adenocarcinoma infected lung tissues using RNA-Seq transcriptome profiling. J Virol Methods. 2020;284:113923. https://doi.org/10.1016/j.jviromet.2020.113923.

24. Edmunds RC, McIntyre JK, Luckenbach JA, Baldwin DH, Incardona JP. Toward enhanced MIQE compliance: reference residual normalization of qPCR gene expression data. J Biomol Tech. 2014;25(2):54-60. https://doi.org/10.7171/jbt.14-2502-003.

25. Zhou C, Liang Y, Zhou L, Yan Y, Liu N, Zhang R, Huang Y, Wang M, Tang Y, Ali DW, et al. TSPAN1 promotes autophagy flux and mediates cooperation between WNT-CTNNB1 signaling and autophagy via the MIR454-FAM83A-TSPAN1 axis in pancreatic cancer. Autophagy. 2021;17(10):3175-3195. https://doi.org/10.1080/15548627.2020.1826689.

26. Zhang Y, Shi J, Liu S. Establishment and Characterization of a Telomerase-Immortalized Sheep Trophoblast Cell Line. Biomed Res Int. 2016;2016:5808575. https://doi.org/10.1155/2016/5808575.

27. Jin Y, Li Y, Wang X, Yang Y. Secretory leukocyte protease inhibitor suppresses HPV E6-expressing HNSCC progression by mediating NF- $\mathrm{kB}$ and Akt pathways. Cancer Cell Int. 2019;19:220. https://doi.org/10.1186/s12935-019-0942-7.

28. Wang B, Lan T, Xiao H, Chen ZH, Wei C, Chen LF, Guan JF, Yuan RF, Yu X, Hu $\mathrm{ZG}$, at al. The expression profiles and prognostic values of HSP70s in hepatocellular carcinoma. Cancer Cell Int. 2021;21(1):286. https://doi.org/10.1186/s12935-021-01987-9

29. Choudhury M, Yin X, Schaefbauer KJ, Kang JH, Roy B, Kottom TJ, Limper AH, Leof EB. SIRT7-mediated modulation of glutaminase 1 regulates TGF- $\beta$-induced 
pulmonary fibrosis. FASEB J. 2020;34(7):8920-8940.

https://doi.org/10.1096/fj.202000564R.

30. Zuo J, Yu Y, Zhu M, Jing W, Yu M, Chai H, Liang C, Tu J. Inhibition of miR-155 a therapeutic target for breast cancer prevented in cancer stem cell formation. Cancer Biomark. 2018;21(2):383-392. https://doi.org/10.3233/CBM-170642.

31. Wang P, Gong Y, Guo T, Li M, Fang L, Yin S, Kamran M, Liu Y, Xu J, Xu L, et al. Activation of Aurora A kinase increases YAP stability via blockage of autophagy. Cell Death Dis. 2019;10(6):432. https://doi.org/10.1038/s41419-019-1664-4.

32. Chen HY, Shen H, Jia B, Zhang YS, Wang XH, Zeng XC. Differential gene expression in ovaries of Qira black sheep and Hetian sheep using RNA-Seq technique. PLoS One. 2015;10(3):e0120170. https://doi.org/10.1371/journal.pone.0120170.

33. Wesche J, Haglund K, Haugsten EM. Fibroblast growth factors and their receptors in cancer. Biochem J. 2011;437(2):199-213. https://doi.org/10.1042/BJ20101603.

34. Petropoulos M, Champeris Tsaniras S, Taraviras S, Lygerou Z. Replication Licensing Aberrations Replication Stress and Genomic Instability. Trends Biochem Sci. 2019;44(9):752-764. https://doi.org/10.1016/j.tibs.2019.03.011.

35. da Silva Sergio LP, Mencalha AL, de Souza da Fonseca A, de Paoli F. DNA repair and genomic stability in lungs affected by acute injury. Biomed Pharmacother. 2019;119:109412. https://doi.org/10.1016/j.biopha.2019.109412.

36. Riera-Domingo C, Audigé A, Granja S, Cheng WC, Ho PC, Baltazar F, Stockmann C, Mazzone M. Immunity Hypoxia and Metabolism-the Ménage à Trois of Cancer: Implications for Immunotherapy. Physiol Rev. 2020;100(1):1-102. https://doi.org/10.1152/physrev.00018.2019.

37. Warbrick I, Rabkin SW. Hypoxia-inducible factor 1-alpha (HIF-1 $\alpha$ ) as a factor mediating the relationship between obesity and heart failure with preserved ejection fraction. Obes Rev. 2019;20(5):701-712. https://doi.org/10.1111/obr.12828.

38. Zhao Y, Hu Q, Cheng F, Su N, Wang A, Zou Y, Hu H, Chen X, Zhou HM, Huang $X$, et al. SoNar a Highly Responsive NAD+/NADH Sensor Allows High-Throughput Metabolic Screening of Anti-tumor Agents. Cell Metab. 2015;21(5):777-89. https://doi.org/10.1016/j.cmet.2015.04.009.

39. Maeda N, Fu W, Ortin A, De las Heras M, Fan H. Roles of the Ras-MEK-mitogen-activated protein kinase and phosphatidylinositol 3-kinase-Akt-mTOR pathways in Jaagsiekte sheep retrovirus-induced transformation of rodent fibroblast and epithelial cell lines. J Virol. 2005;79(7):4440-4450. https://doi.org/10.1128/JVI.79.7.4440-4450.2005.

40. Gao S, Ni Q, Wu X, Cao T. GHR knockdown enhances the sensitivity of HCC cells to sorafenib. Aging (Albany NY). 2020;12(18):18127-18136. https://doi.org/10.18632/aging.103625.

41. Wu X, Li M, Li Y, Deng Y, Ke S, Li F, Wang Y, Zhou S. Fibroblast growth factor 11 (FGF11) promotes non-small cell lung cancer (NSCLC) progression by regulating hypoxia signaling pathway. J Transl Med. 2021;19(1):353. https://doi.org/10.1186/s12967-021-03018-7. 
42. Justice RW, Zilian O, Woods DF, Noll M, Bryant PJ. The Drosophila tumor suppressor gene warts encodes a homolog of human myotonic dystrophy kinase and is required for the control of cell shape and proliferation. Genes Dev. 1995;9(5):534-546. https://doi.org/10.1101/gad.9.5.534.

43. Stewart RA, Li DM, Huang H, Xu T. A genetic screen for modifiers of the lats tumor suppressor gene identifies C-terminal Src kinase as a regulator of cell proliferation in Drosophila. Oncogene. 2003;25;22(41):6436-6444. https://doi.org/10.1038/sj.onc.1206820.

44. Zhao B, Lei QY, Guan KL. The Hippo-YAP pathway: new connections between regulation of organ size and cancer. Curr Opin Cell Biol. 2008 Dec;20(6):638-46. https://doi.org/10.1016/j.ceb.2008.10.001.

45. Zhao B, Wei X, Li W, Udan RS, Yang Q, Kim J, Xie J, Ikenoue T, Yu J, Li L, et al. Inactivation of YAP oncoprotein by the Hippo pathway is involved in cell contact inhibition and tissue growth control. Genes Dev. 2007;21(21):2747-2761. https://doi.org/10.1101/gad.1602907.

46. Matallanas D, Romano D, Hamilton G, Kolch W, O'Neill E. A Hippo in the ointment: MST signalling beyond the fly. Cell Cycle. 2008;7(7):879-884. https://doi.org/10.4161/cc.7.7.5630.

47. Callus BA, Verhagen AM, Vaux DL. Association of mammalian sterile twenty kinases Mst1 and Mst2 with hSalvador via C-terminal coiled-coil domains leads to its stabilization and phosphorylation. FEBS J. 2006;273(18):4264-4276. https://doi.org/10.1111/j.1742-4658.2006.05427.x.

48. Chan EH, Nousiainen M, Chalamalasetty RB, Schäfer A, Nigg EA, Silljé HH. The Ste20-like kinase Mst2 activates the human large tumor suppressor kinase Lats1. Oncogene. 2005;24(12):2076-2086. https://doi.org/10.1038/sj.onc.1208445.

49. Praskova M, Xia F, Avruch J. MOBKL1A/MOBKL1B phosphorylation by MST1 and MST2 inhibits cell proliferation. Curr Biol. 2008;18(5):311-321. https://doi.org/10.1016/j.cub.2008.02.006.

50. Huang J, Wu S, Barrera J, Matthews K, Pan D. The Hippo signaling pathway coordinately regulates cell proliferation and apoptosis by inactivating Yorkie the Drosophila Homolog of YAP. Cell. 2005;122(3):421-434. https://doi.org/10.1016/j.cell.2005.06.007.

51. Zhao B, Li L, Tumaneng K, Wang CY, Guan KL. A coordinated phosphorylation by Lats and CK1 regulates YAP stability through SCF(beta-TRCP). Genes Dev. 2010;24(1):72-85. https://doi.org/10.1101/gad.1843810.

52. Kim MK, Jang JW, Bae SC. DNA binding partners of YAP/TAZ. BMB Rep. 2018;51(3):126-133. https://doi.org/10.5483/bmbrep.2018.51.3.015.

53. Zhao B, Ye X, Yu J, Li L, Li W, Li S, Yu J, Lin JD, Wang CY, Chinnaiyan AM, et al. TEAD mediates YAP-dependent gene induction and growth control. Genes Dev. 2008;22(14):1962-71. https://doi.org/10.1101/gad.1664408.

54. Ferrigno O, Lallemand F, Verrecchia F, L'Hoste S, Camonis J, Atfi A, Mauviel A. Yes-associated protein (YAP65) interacts with Smad7 and potentiates its inhibitory activity against TGF-beta/Smad signaling. Oncogene. 2002;21(32):4879-4884. https://doi.org/10.1038/sj.onc.1205623. 
55. Wang Y, Dong Q, Zhang Q, Li Z, Wang E, Qiu X. Overexpression of yes-associated protein contributes to progression and poor prognosis of non-small-cell lung cancer. Cancer Sci. 2010;101(5):1279-1285. https://doi.org/10.1111/j.1349-7006.2010.01511.X.

56. Xu W, Zhang M, Li Y, Wang Y, Wang K, Chen Q, Zhang R, Song W, Huang Q, Zhao W, et al. YAP manipulates proliferation via PTEN/AKT/mTOR-mediated autophagy in lung adenocarcinomas. Cancer Cell Int. 2021;21(1):30. https://doi.org/10.1186/s12935-020-01688-9.

57. Dong J, Feldmann G, Huang J, Wu S, Zhang N, Comerford SA, Gayyed MF, Anders RA, Maitra A, Pan D. Elucidation of a universal size-control mechanism in Drosophila and mammals. Cell. 2007;130(6):1120-1133. https://doi.org/10.1016/j.cell.2007.07.019.

58. Sawada A, Kiyonari H, Ukita K, Nishioka N, Imuta Y, Sasaki H, Redundant roles of Tead 1 and Tead 2 in notochord development and the regulation of cell proliferation and survival. Mol Cell Biol. 2008;28(10):3177-3189. https://doi.org/10.1128/MCB.01759-07.

59. Zhou D, Conrad C, Xia F, Park JS, Payer B, Yin Y, Lauwers GY, Thasler W, Lee JT, Avruch J, et al. Mst1 and Mst2 maintain hepatocyte quiescence and suppress hepatocellular carcinoma development through inactivation of the Yap1 oncogene. Cancer Cell. 2009;16(5):425-438. https://doi.org/10.1016/j.ccr.2009.09.026.

60. Xu MZ, Yao TJ, Lee NP, Ng IO, Chan YT, Zender L, Lowe SW, Poon RT, Luk JM. Yes-associated protein is an independent prognostic marker in hepatocellular carcinoma. Cancer. 2009;115(19):4576-4585. https://doi.org/10.1002/cncr.24495.

61. Dong A, Gupta A, Pai RK, Tun M, Lowe AW. The human adenocarcinoma-associated gene, AGR2, induces expression of amphiregulin through Hippo pathway co-activator YAP1 activation. J Biol Chem. 2011;286(20):18301-18310. https://doi.org/10.1074/jbc.M110.215707.

62. Steinhardt AA, Gayyed MF, Klein AP, Dong J, Maitra A, Pan D, Montgomery EA, Anders RA. Expression of Yes-associated protein in common solid tumors. Hum Pathol. 2008;39(11):1582-1589. https://doi.org/10.1016/j.humpath.2008.04.012.

63. Yu DL, Chow N, Wootton SK. JSRV Intragenic Enhancer Element Increases Expression from a Heterologous Promoter and Promotes High Level AAV-mediated Transgene Expression in the Lung and Liver of Mice. Viruses. 2020;12(11):1266. https://doi.org/10.3390/v12111266.

64. Palmarini M, Datta S, Omid R, Murgia C, Fan H. The long terminal repeat of Jaagsiekte sheep retrovirus is preferentially active in differentiated epithelial cells of the lungs. J Virol. 2000;74(13):5776-5787. https://doi.org/10.1128/jvi.74.13.5776-5787.2000.

65. Archer F, Jacquier E, Lyon M, Chastang J, Cottin V, Mornex JF, Leroux C. Alveolar type II cells isolated from pulmonary adenocarcinoma: a model for JSRV expression in vitro. Am J Respir Cell Mol Biol. 2007;36(5):534-540. https://doi.org/10.1165/rcmb.2006-0285OC.

66. Moroishi T, Park HW, Qin B, Chen Q, Meng Z, Plouffe SW, Taniguchi K, Yu FX, Karin M, Pan D, et al. A YAP/TAZ-induced feedback mechanism regulates Hippo 
pathway homeostasis. Genes Dev. 2015;29(12):1271-1284.

https://doi.org/10.1101/gad.262816.115.

67. Park GS, Oh H, Kim M, Kim T, Johnson RL, Irvine KD, Lim DS. An evolutionarily conserved negative feedback mechanism in the Hippo pathway reflects functional difference between LATS1 and LATS2. Oncotarget. 2016;7(17):24063-24075. https://doi.org/10.18632/oncotarget.8211.

68. Moroishi T, Hayashi T, Pan WW, Fujita Y, Holt MV, Qin J, Carson DA, Guan KL. The Hippo Pathway Kinases LATS1/2 Suppress Cancer Immunity. Cell. 2016;167(6):1525-1539.e17. https://doi.org/10.1016/j.cell.2016.11.005.

69. Gabriel BM, Hamilton DL, Tremblay AM, Wackerhage H. The Hippo signal transduction network for exercise physiologists. J Appl Physiol (1985). 2016;120(10):1105-1117. https://doi.org/10.1152/japplphysiol.01076.2015.

\section{Figure Legends}

Figure 1. Gene expression profiles of naturally infected OPA cases and healthy cases. (a) Principal component analysis of DEGs in naturally infected OPA cases and healthy cases. (b) Volcano plot of DEGs in naturally infected OPA cases and healthy cases. The red dots represent the 154 upregulated genes; the blue dots represent the 212 downregulated genes; the gray dots represent the unchanged genes. (c) Hierarchical cluster diagram of the top 100 differentially expressed transcripts of the DEGs in naturally infected OPA cases based on log10-transformed expression values (fragments per kilobase of transcript per million fragments mapped FPKM). Y-axis shows the list of DEGs and $\mathrm{X}$-axis shows control and samples.

Figure 2. Bubble plots comparing GO enrichment and KEGG pathway analysis. (a) The 20 most significantly enriched GO terms for DEGs. (b) The 20 most significantly enriched KEGG pathways for DEGs.

Figure 3. RT-qPCR and RNA-Seq of 10 DEGs. The relative expression level (FC)of a mRNA transcript refers to the change in expression of naturally infected OPA cases relative healthy sheep determined by using the $2^{-\Delta \Delta C T}$ method.

Figure 4. The detection of Hippo signaling pathway core components proteins in lung of naturally infected OPA cases and healthy sheep. (A) Immunohistochemical detection of lung sections. Left column, lung of healthy sheep; right column, lung of naturally infected OPA cases. Brown pigment indicates positive labeling. (a to b) negative control; (c to d) JSRV Env; (e to f) MST1/2; 

was strongly positive in nucleus and cytoplasm of proliferative type II pneumocytes and nuclear

881 labeling was more intense than the labeling of the cytoplasm (j). The phosphorylated YAP1 was predominantly detected in the cytoplasm of proliferative type II pneumocytes (1). Red arrows in panels $\mathrm{j}$ indicate YAP1 locating in the nucleus. (B) Results are the mean of four separate experiments performed in triplicate \pm SD. $p<0.05$ significantly different from healthy control groups. (C) Western blot analysis of Hippo signaling pathway core components in lung of naturally infected OPA cases and healthy sheep. (D)The data are presented as mean \pm SD. value from the lungs of 3 healthy sheep and 3 naturally infected OPA cases $(p<0.05)$.

Figure 5. The detection of biological characteristics and western blot analysis of Hippo pathway components (MST1/2 LATS1/2 YAP1 and p-YAP1). (A) (a) uninfected STCs in the bright field; (b)uninfected STCs in the fluorescence field; (c) empty virus vector infected STCs in the bright field; (d) empty virus vector infected STCs in the fluorescence field; (e) JSRV-env lentivirus infected STCs in the bright field; (f) JSRV-env lentivirus infected STCs in the fluorescence field. (B) The data were presented as mean \pm SD. value from 3 blank groups 3 negative control groups and 3 experimental groups $(p<0.0001)$. (C) The cell proliferation rate was evaluated in JSRV Env-transformed STCs and control groups by the MTT assay $(p<0.01)$. (D) The migration rate of cells was detected in JSRV Env-transformed STCs and control groups by wound-healing assay. (E) The data of scratch areas were presented as mean \pm SD. value from 3 blank groups, 3 negative control groups, 3 positive control groups and 3 experimental groups $(p<0.001)$. (F) The invasion rate of cells was detected in JSRV Env-transformed STCs and control groups by transwell assay. (G) The data of transwell assay were presented as mean \pm SD. value from 3 blank groups, 3 negative control groups, 3 positive control groups and 3 experimental groups $(p<0.0001)$. (H) Soft agar colony formation assay of JSRV Env-transformed STCs and control groups. (I) Western blot analysis of Hippo pathway components (MST1/2 LATS1/2 YAP1 and p-YAP1) in JSRV Env-transformed STCs and control groups. (D) The data were presented as mean \pm SD. value from 3 blank groups 3 negative control groups and 3 experimental groups $(p<0.05)$. 
Figures

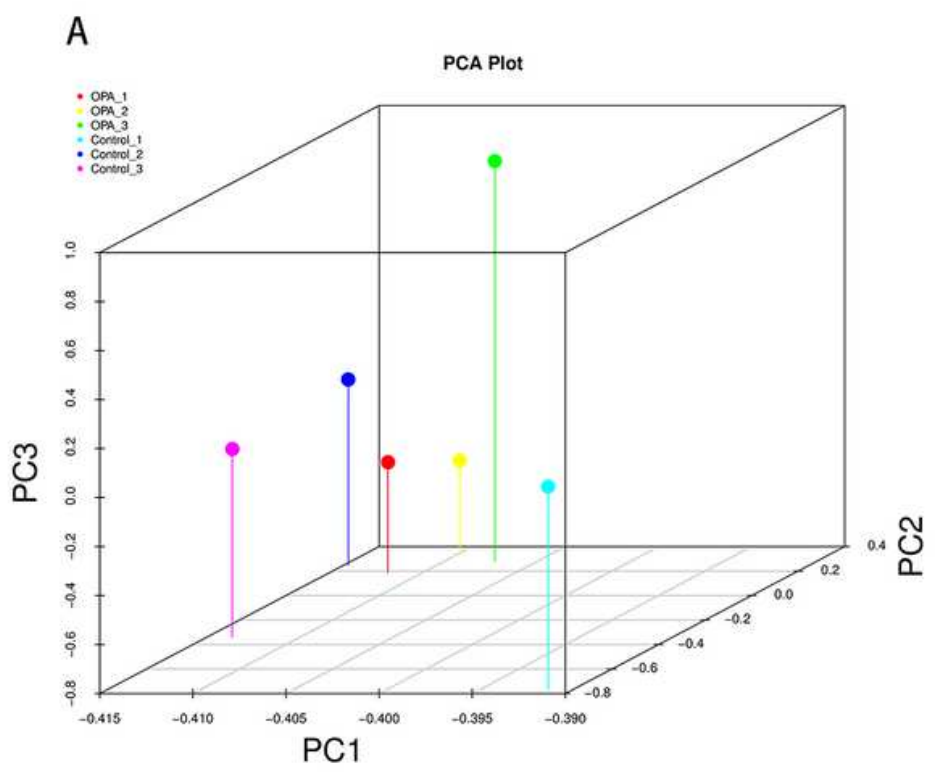

B opavscontrol

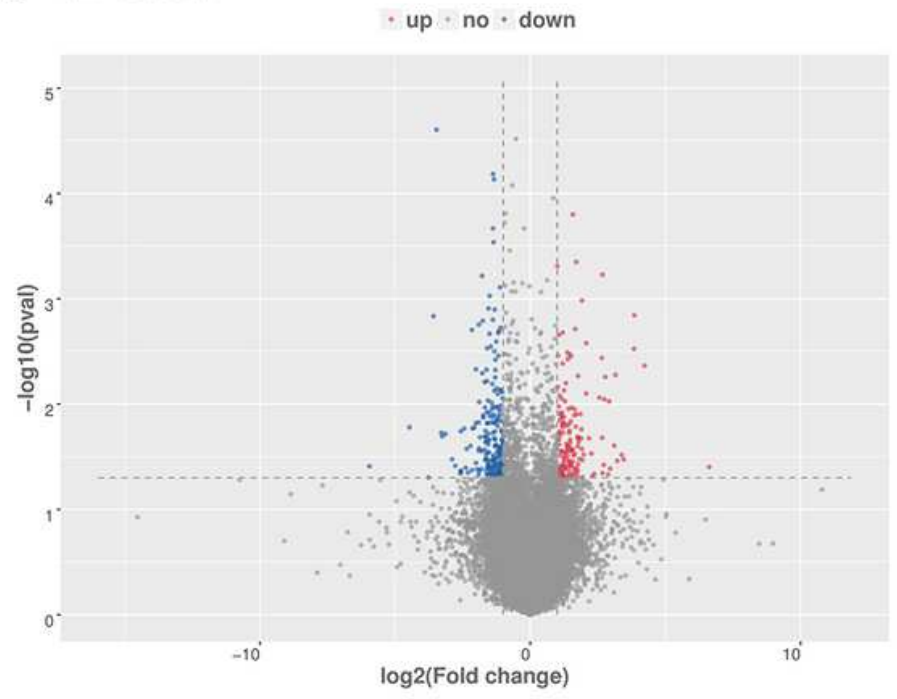

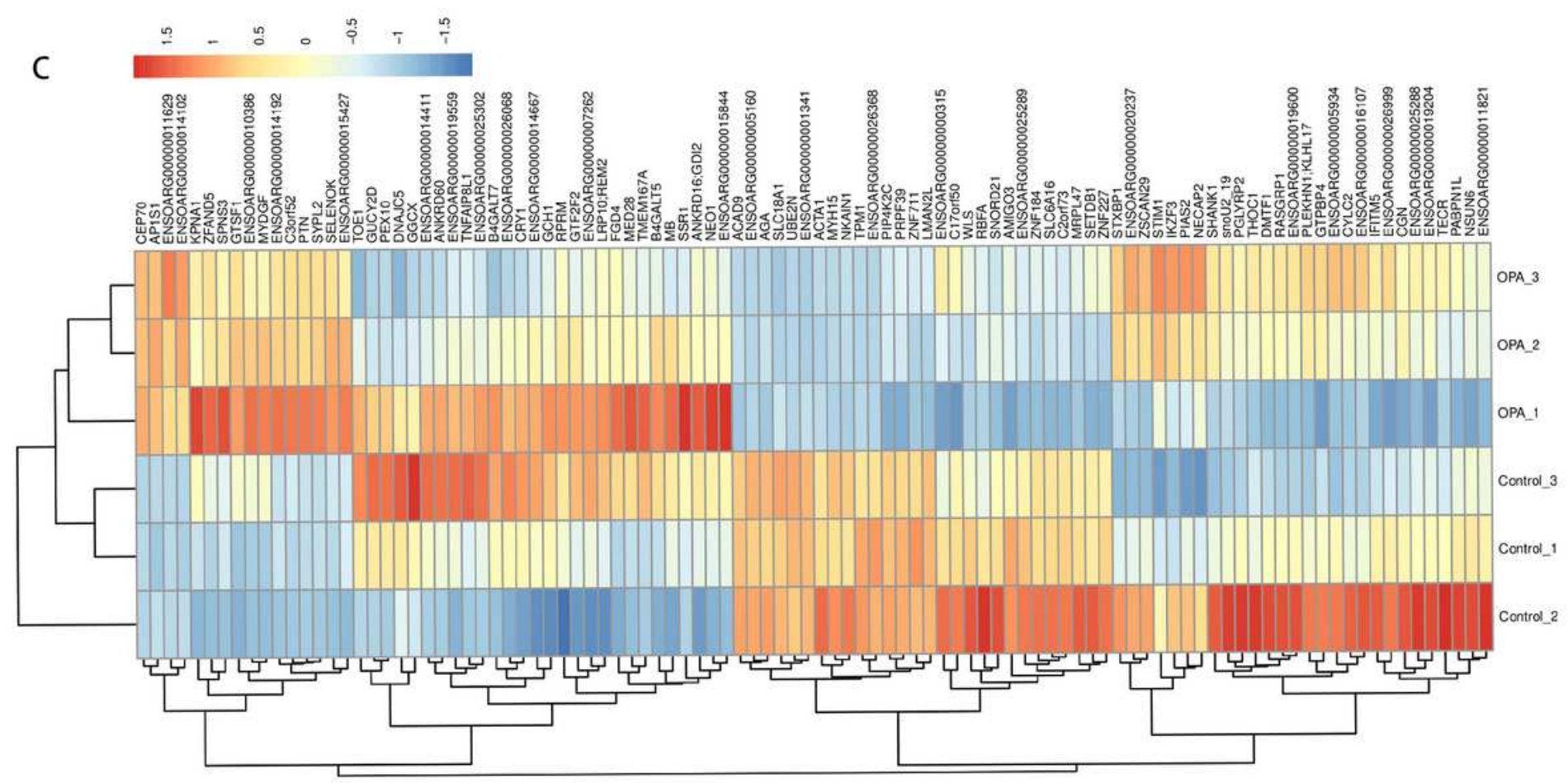

Figure 1

Gene expression profiles of naturally infected OPA cases and healthy cases (a) Principal component analysis of DEGs in naturally infected OPA cases and healthy cases (b) Volcano plot of DEGs in naturally infected OPA cases and healthy cases. The red dots re present the 154 upregulated genes; the blue dots represent the 212 downregulated genes; the gray dots represent the unchanged genes. (c) Hierarchical cluster diagram of the top 100 differentially expressed transcripts of the DEGs in naturally infected OPA 
cases based on log10-transformed expression values (fragments per kilobase of transcript per million fragments mapped FPKM) Y-axis shows the list of DEGs and X-axis shows control and sample s.

A

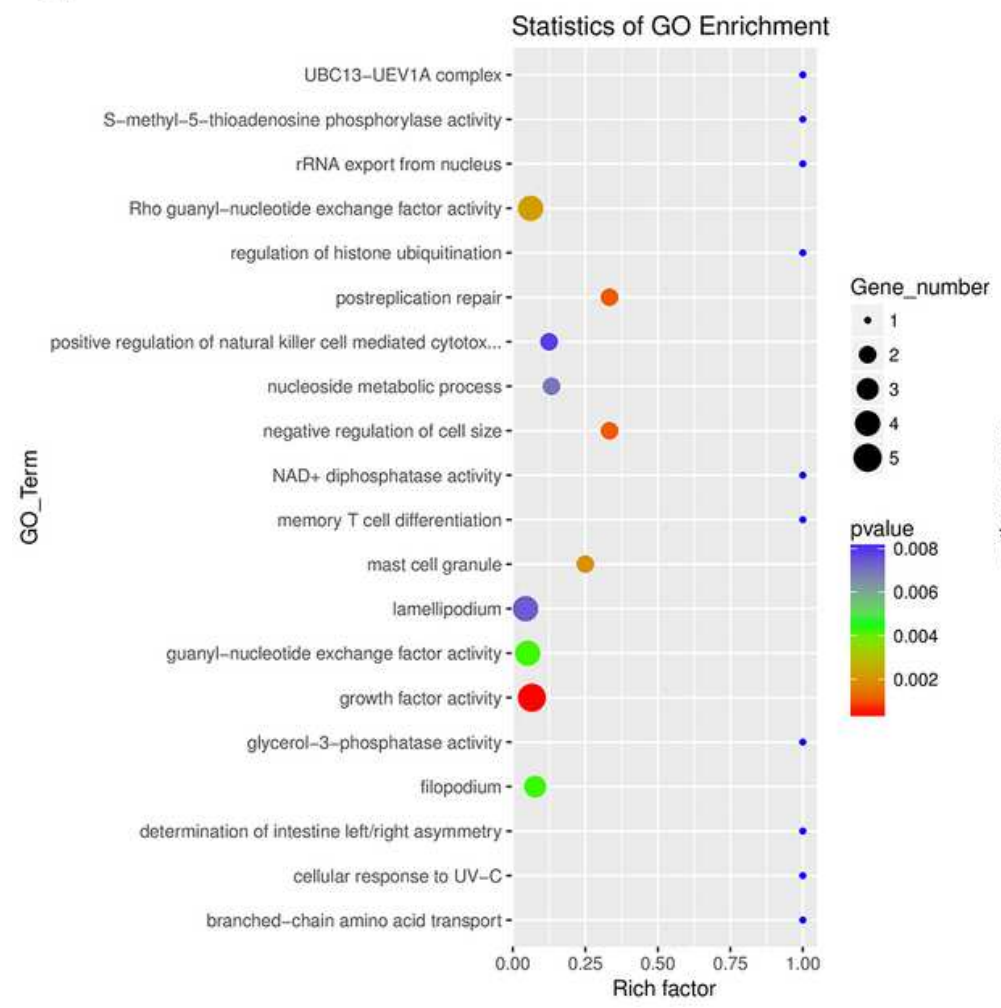

B

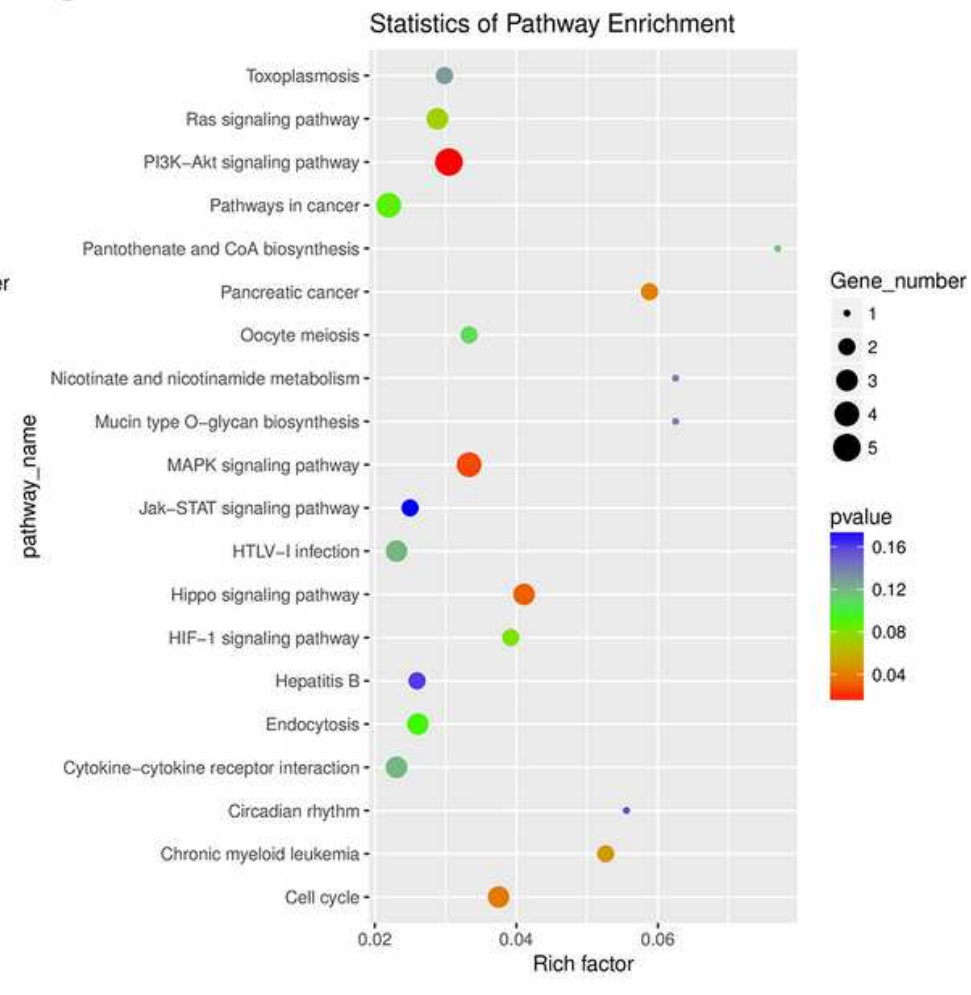

\section{Figure 2}

Bubble plots co mparing GO enrichment and KEGG pathw ay analysis. (a) The 20 most significantly enriched GO terms for DEGs. (b) The 20 most signific antly enriched K EGG pathwa ys for DEGs. 


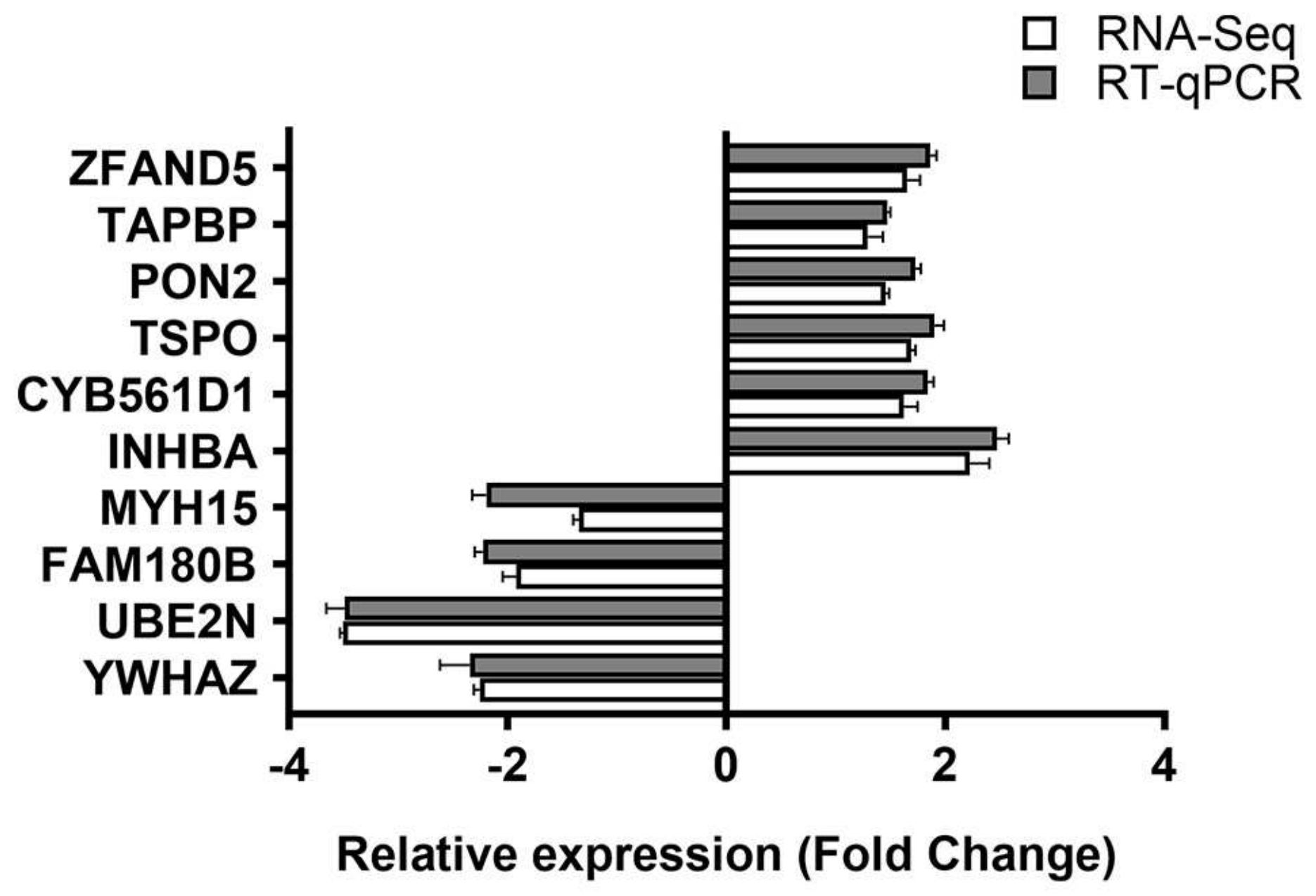

Figure 3

RT-qPCR an d RNA-Seq of 10 DEGs. The relative expression level (FC) of a mRNA transcript refers to the change in expression of naturally infected OPA cases relative healthy sheep determined by using the $2 \mathrm{CT}$ method. 
A
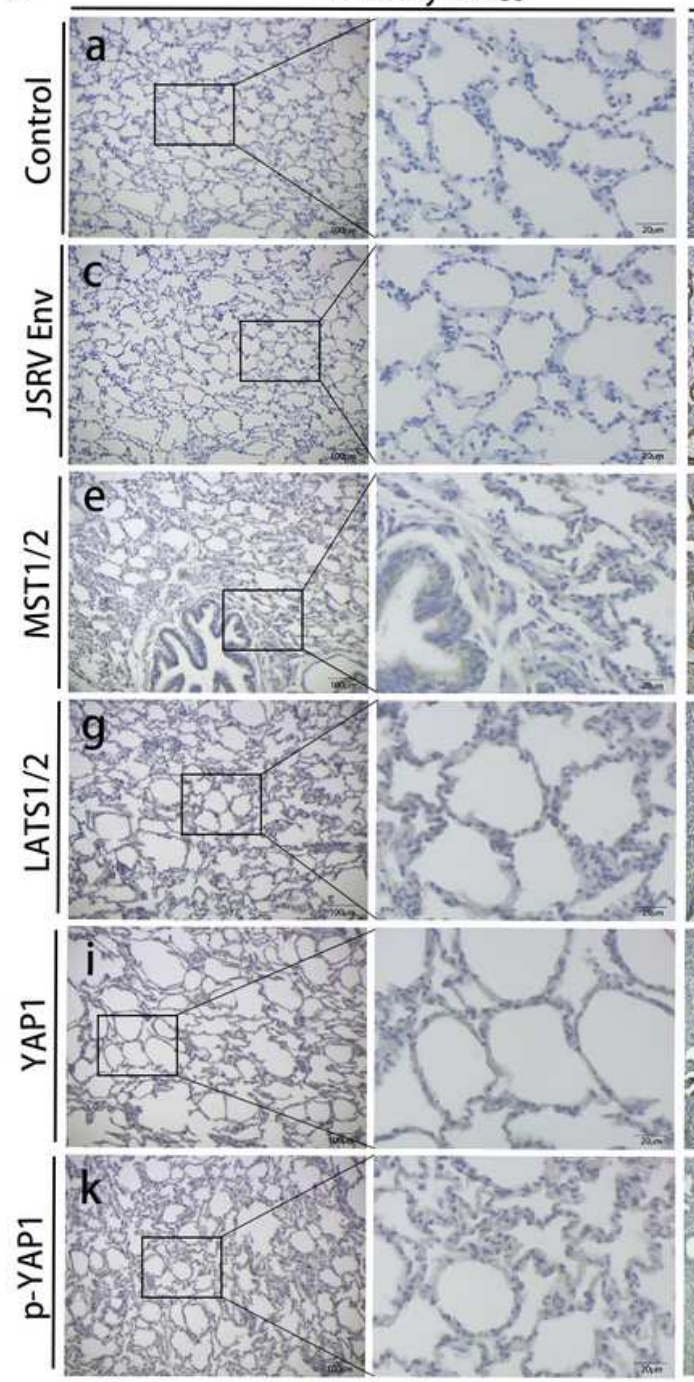

OPA cases
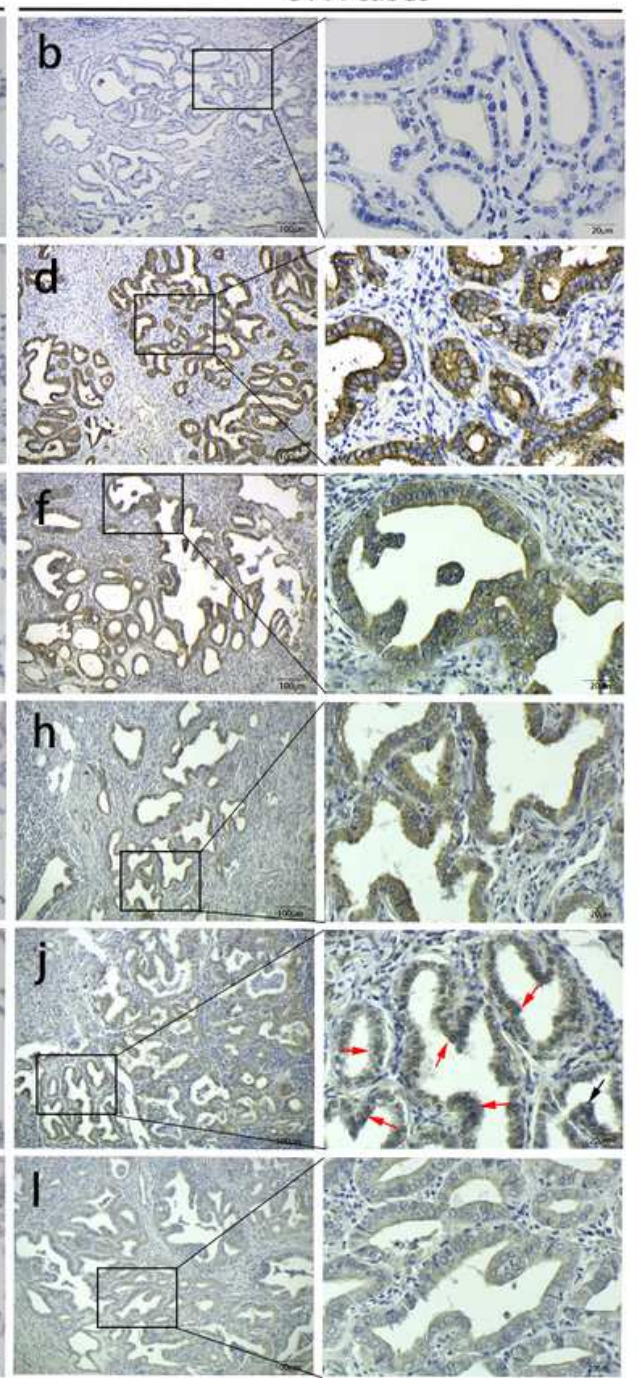

C
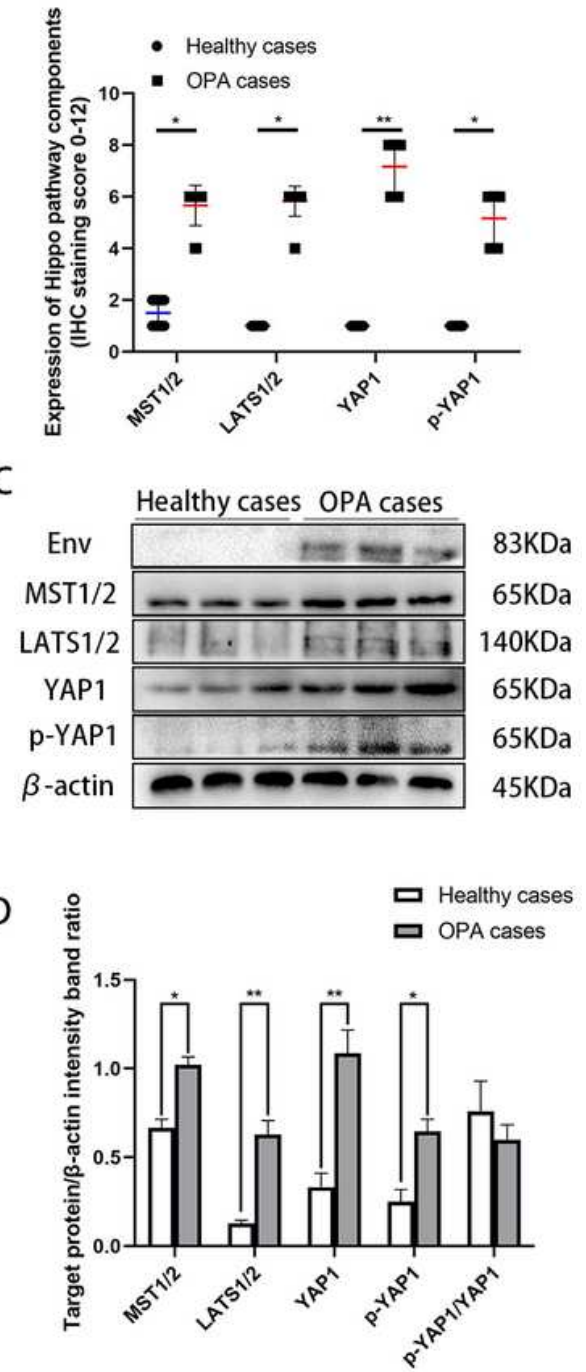

Figure 4

The detection of Hippo signaling pathway core components proteins in lung of naturally infected OPA cases and healthy sheep. (A) Immunohistochemical detection of lung sections. Left column, lung of healthy sheep; right column, lung of naturally infected OPA cases. Brown pigment indicates positive labeling. ( $a$ to b) negative control; (c to d) JSRV Env; (e to f) MST1/2; 3 ( $g$ to h) LATS1/2; ( $\mathrm{i}$ to j) Total YAP1; ( $k$ to l) phosphorylated YAP1 (p-YAP1). Note that YAP1 was strongly positive in nucleus and cytoplasm of proliferative type II pneumocytes and nuclear labeling was more intense than the labeling of the cytoplasm (j). The phosphorylated YAP1 was predominantly detected in the cytoplasm of proliferative type II pneumocytes (I). Red arrows in panels $\mathrm{j}$ indicate YAP1 locating in the nucleus. (B) Results are the mean of four separate experiments performed in triplicate \pm SD. $p<0.05$ significantly different from healthy control groups. (C) Western blot analysis of Hippo signaling pathway core components in lung of naturally infected OPA cases and healthy sheep. (D)The data are presented as mean \pm SD. value from the lungs of 3 healthy sheep and 3 naturally infected OPA cases $(p<0.05)$. 

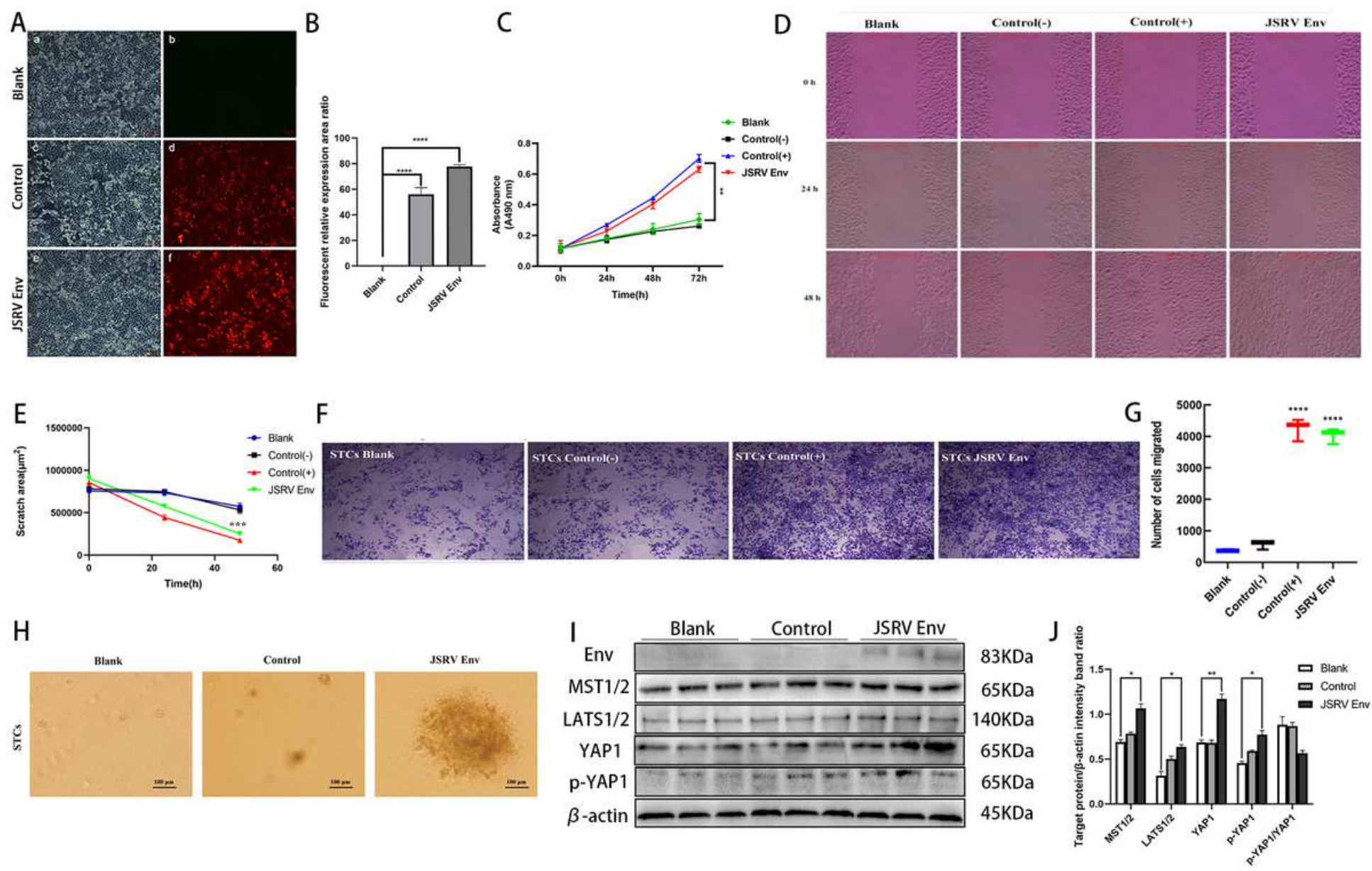

\section{Figure 5}

The detection of biological characteristics and western blot analysis of Hippo pathway components (MST1/2 LATS1/2 YAP1 and p-YAP1). (A) (a) uninfected STCs in the bright field; (b)uninfected STCs in the fluorescence field; (c) empty virus vector infected STCs in the bright field; (d) empty virus vector infected STCs in the fluorescence field; (e) JSRV-env lentivirus infected STCs in the bright field; (f) JSRVenv lentivirus infected STCs in the fluorescence field. (B) The data were presented as mean \pm SD. value from 3 blank groups 3 negative control groups control groups, 3 positive control groups and 3 experimental groups $(\mathrm{p}<0.001)$. ( $F)$ The invasion rate of cells was detected in JSRV Env-transformed STCs and control groups by transwell assay. (G) The data of transwell assay were presented as mean $\pm S D$. value from 3 blank groups, 3 negative control groups, 3 positive control groups and 3 experimental groups $(p<0.0001)$. $(H)$ Soft agar colony formation assay of JSRV Env-transformed STCs and control groups. (I) Western blot analysis of Hippo pathway components (MST1/2 LATS1/2 YAP1 and p-YAP1) in JSRV Env-transformed STCs and control groups. (D) The data were presented as mean \pm SD. value from 3 blank groups 3 negative control groups and 3 experimental groups $(p<0.05)$. 